\title{
How to describe a cryptic species? Practical challenges of molecular taxonomy
}

\author{
Katharina M Jörger ${ }^{1,2^{*}}$ and Michael Schrödl ${ }^{1,2}$
}

\begin{abstract}
Background: Molecular methods of species delineation are rapidly developing and widely considered as fast and efficient means to discover species and face the 'taxonomic impediment' in times of biodiversity crisis. So far, however, this form of DNA taxonomy frequently remains incomplete, lacking the final step of formal species description, thus enhancing rather than reducing impediments in taxonomy. DNA sequence information contributes valuable diagnostic characters and -at least for cryptic species - could even serve as the backbone of a taxonomic description. To this end solutions for a number of practical problems must be found, including a way in which molecular data can be presented to fulfill the formal requirements every description must meet. Multi-gene barcoding and a combined molecular species delineation approach recently revealed a radiation of at least 12 more or less cryptic species in the marine meiofaunal slug genus Pontohedyle (Acochlidia, Heterobranchia). All identified candidate species are well delimited by a consensus across different methods based on mitochondrial and nuclear markers.

Results: The detailed microanatomical redescription of Pontohedyle verrucosa provided in the present paper does not reveal reliable characters for diagnosing even the two major clades identified within the genus on molecular data. We thus characterize three previously valid Pontohedyle species based on four genetic markers (mitochondrial cytochrome c oxidase subunit I, 165 rRNA, nuclear 285 and $18 \mathrm{~S}$ rRNA) and formally describe nine cryptic new species (P. kepii sp. nov., P. joni sp. nov., P. neridae sp. nov., P. liliae sp. nov., P. wiggi sp. nov., P. wenzli sp. nov., P. peteryalli sp. nov., P. martynovi sp. nov., P. yurihookeri sp. nov.) applying molecular taxonomy, based on diagnostic nucleotides in DNA sequences of the four markers. Due to the minute size of the animals, entire specimens were used for extraction, consequently the holotype is a voucher of extracted DNA ('DNA-type'). We used the Character Attribute Organization System (CAOS) to determine diagnostic nucleotides, explore the dependence on input data and data processing, and aim for maximum traceability in our diagnoses for future research. Challenges, pitfalls and necessary considerations for applied DNA taxonomy are critically evaluated.

Conclusions: To describe cryptic species traditional lines of evidence in taxonomy need to be modified. DNA sequence information, for example, could even serve as the backbone of a taxonomic description. The present contribution demonstrates that few adaptations are needed to integrate into traditional taxonomy novel diagnoses based on molecular data. The taxonomic community is encouraged to join the discussion and develop a quality standard for molecular taxonomy, ideally in the form of an automated final step in molecular species delineation procedures.
\end{abstract}

\footnotetext{
* Correspondence: Katharina.Joerger@zsm.mwn.de

${ }^{1}$ Mollusca Section, SNSB-Bavarian State Collection of Zoology,

Münchhausenstr 21, 81247 München, Germany

${ }^{2}$ Department Biology II, Ludwig-Maximilians-University, Großhaderner Str. 2,

82152 Planegg-Martinsried, Germany
}

\section{( Biomed Central}

(c) 2013 Jörger and Schrödl; licensee BioMed Central Ltd. This is an Open Access article distributed under the terms of the Creative Commons Attribution License (http://creativecommons.org/licenses/by/2.0), which permits unrestricted use, distribution, and reproduction in any medium, provided the original work is properly cited. 


\section{Background}

Species boundaries are frequently hard to delimit based on morphology only, a fact which has called for integrative taxonomy, including additional sources of information such as molecular data, biogeography, behavior and ecology [1,2]. Founding a species description on a variety of characters from different, independent datasets is generally regarded as best practice [3]. When species are considered as independently evolving lineages [4], different lines of evidence (e.g., from morphology, molecules, ecology or distribution) are additive to each other and no line is necessarily exclusive nor need different lines obligatory be used in combination $[3,5]$. Taxonomists are urged to discriminate characters according to their quality and suitability for species delineation, rather than to just add more and more data [5]. The specifics of the taxon in question will guide the way to the respective set(s) of characters that will provide the best backbone for the diagnosis. In cases of pseudo-cryptic species (among which morphological differences can be detected upon re-examining lineages separated e.g. on molecular data) or of fully cryptic species (that morphology fails to delimit), the traditional lines of evidence have to be modified by using, e.g., molecular information to break out of the 'taxonomic circle' [6,7].

Cryptic species are a common phenomenon throughout the metazoan taxa, and can be found in all sorts of habitats and biogeographic zones [8-10]. Groups characterized by poor dispersal abilities (e.g., most meiofaunal organisms or animals inhabiting special regions where direct developers predominate, such as Antarctica), are especially prone to cryptic speciation $[11,12]$. Uncovering these cryptic species is fundamental for the understanding of evolutionary processes, historical biogeography, ecology, and also to conservation approaches, as distribution ranges that are smaller than initially assumed mean a higher risk of local extinction $[8,10]$. The lack of morphological characters to distinguish cryptic species should not lead to considerable parts of biological diversity remaining unaddressed.

The utility of DNA barcoding and molecular species delineation approaches to uncover cryptic lineages has been demonstrated by numerous studies (e.g., [11,13-19]). Unfortunately, inconsistencies in terminology associated with the interface between sequence data and taxonomy have led to confusion and various criticisms [6,20]. First of all, one needs to distinguish between species identification via molecular data (DNA barcoding in its strict sense) and species discovery $[6,21,22]$. While species identification is a primary technical application, species delimitation requires means of molecular species delineation that is either distance, tree or character based $[6,23]$. Under ideal circumstances sufficient material is collected from different populations across the entire distribution area of a putative group of cryptic species. Using population genetics the distribution of haplotypes can be analyzed and different, genetically isolated lineages can be detected [24]. Population genetic approaches are, however, not always feasible with animals that are rare or hard to collect, which might actually be a common phenomenon across faunas of most marine ecosystems [25-28]. Derived from barcoding initiatives, threshold based species delimitation became the method of choice, aiming for the detection of a 'barcoding gap' between intraand interspecific variation [29-31]. This approach has been criticized, however, due to its sensitivity to the degree of sampling, the general arbitrariness of fixed or relative thresholds, and to frequent overlap between intra- and interspecific variation $[6,32,33]$. In the recently developed Automatic Barcode Gap Discovery (ABGD) [34], progress has been made in avoiding the dependence of a priori defined species hypotheses in threshold based approaches, but reservations remain concerning the concept of a barcoding gap [25]. Several independent delineation tools exist, e.g. using haplotype networks based on statistical parsimony [35], maximum likelihood approaches applying the General Mixed Yule-Coalescent model [36,37], or Bayesian species delineation $[38,39]$. Empirical research currently compares the powers of these different tools on real datasets $[25,32,40]$. The effect of the inclusion of singletons in analyses is considered as most problematic [25]. At the present stage of knowledge, independent approaches allowing cross-validation between the different methods of molecular species delineation and other sources of information (morphology, biogeography, behavioral traits) seem the most reliable way of delimiting cryptic species [25].

The second inconsistency in terminology concerns usages of 'DNA taxonomy'. Originally, DNA taxonomy was proposed to revolutionize taxonomy by generally founding descriptions on sequence data and overthrowing the Linnaean binominal system [41]. Alternatively, it was suggested as a concept of clustering DNA barcodes into MOTUs [42]. Since then, however, it has been applied as an umbrella term for barcoding, molecular species delineation, and including molecular data in species descriptions (see e.g., $[13,14,20,36,43,44])$. In a strict sense, one cannot speak of molecular taxonomy if the process of species discovery is not followed by formal species description (i.e. there are two steps to a taxonomic process: species discovery (delimitation) and attributing them with formal diagnoses and names.) Taxonomy remains incomplete if species hypotheses new to science are flagged as merely putative by provisional rather than fully established scientific names. For practical reasons and journal requirements, most studies on molecular species delineation postpone formal descriptions of the discovered species (e.g., $[13,14,25,33,36,40,43-46])$, and then rarely carry them out later. DNA barcoding and molecular species delineation are promoted as fast and efficient ways to face the 'taxonomic impediment', i.e. the shortage of time and personnel capable of working through the undescribed 
species richness in the middle of a biodiversity crisis $[7,47,48]$. However, keeping discovered entities formally unrecognized does not solve the taxonomic challenges but adds to them by creating parallel worlds populated by numbered MOTUs, OTUs or candidate species. In many cases the discovered taxa remain inapplicable to future research, thus denying the scientific community this taxonomic service, e.g. for species inventories or conservation attempts. Without formal description or a testable hypothesis, i.e. a differential diagnosis, 1) the discovered species might not be properly documented or vouchered by specimens deposited at Natural History Museums; and 2) their reproducibility can be hindered and confusion caused by different numbering systems. A deterrent example of the proliferation of informal epithets circulating as 'nomina nuda' (i.e. species which lack formal diagnoses and deposited vouchers) in the literature is given by the 'ten species in one' Astraptes fulgerator complex [31,49]. Thus, we consider it as all but indispensable for DNA taxonomy to take the final step and formalize the successfully discovered molecular lineages.

The transition from species delimitation to species description is the major task to achieve. Nearly ten years after the original proposal of DNA taxonomy [41], revolutionizing traditional taxonomy has found little acceptance in the taxonomic community, as most authors agree that there is no need for overthrowing the Linnaean System. Consequently, the challenge is to integrate DNA sequence information in the current taxonomic system. Several studies have attempted to include DNA data in taxonomic descriptions, albeit in various non-standardized ways; see the review by Goldstein and DeSalle ([21]; box 3): In some cases, DNA sequence information is simply added to the taxonomic description (in the form of GenBank numbers or pure sequence data), without evaluating and reporting diagnostic features [21]. Others rely on sequence information for the description, either reporting results of species delineation approaches, e.g. raw distance measurements or model based assumptions, or extracting diagnostic characters from their molecular datasets. There still is a consensus that species descriptions should be character based [50] (but see the Discussion below for attempts at model based taxonomy), and that tree or distance based methods fail to extract diagnostic characters [6]. Character based approaches, like the Characteristic Attribute Organization System (CAOS), are suggested as an efficient and reliable way of defining species barcodes based on discrete nucleotide substitution, and these established diagnostics from DNA sequences can be used directly for species descriptions as molecular taxonomic characters [51,52]. Yet, the application of CAOS or similar tools requires an evaluation of how to select and present molecular synapomorphies and how to formalize procedures to create a 'best practice' linking DNA sequence information to existing taxonomy [20].
In the present study, we formally describe the candidate species of minute mesopsammic sea slugs in the genus Pontohedyle Golikov \& Starobogatov (Acochlidia, Heterobranchia) discovered by Jörger et al. [25]. This cryptic radiation was uncovered in a global sampling approach with multi-gene and multiple-method molecular species delineation [25]. The initially identified 12 MOTUs, nine of which do not correspond to described species, are considered as species [following 4] resulting from a conservative minimum consensus approach applying different methods of molecular species delineation [25]. The authors demonstrated that traditional taxonomic characters (external morphology, spicules and radula features) are insufficient to delineate cryptic Pontohedyle species [25]. To evaluate the power of more advanced histological and microanatomical data, we first provide a detailed computer based 3D redescription of the anatomy of Pontohedyle verrucosa (Challis, 1970) and additional histological semithin sections of $P$. kepii sp. nov. In the absence of reliable diagnostic characters from morphology and microanatomy, we then rely on DNA sequence data as the backbone for our species descriptions. For the three previously valid Pontohedyle species we extract diagnostic characters using the Character Attribute Organization System (CAOS) based on four standard markers (mitochondrial cytochrome c oxidase subunit I, $16 \mathrm{~S}$ rRNA, and nuclear $18 \mathrm{~S}$ rRNA and $28 \mathrm{~S}$ rRNA). In addition, nine new species are formally described on molecular characteristics and evidence from other data sources. Various approaches to the practical challenges for molecular driven taxonomy - such as critical consideration of the quality of the alignment, detection of diagnostic nucleotides and their presentation aiming for maximum traceability in future studies - are tested and critically evaluated.

\section{Results}

\section{Evaluation of putative morphological characters}

The diversity within Pontohedyle revealed by molecular data cannot be distinguished externally: the body shows the typical subdivision into the anterior head-foot complex and the posterior visceral hump. Bodies are whitishtranslucent, digestive glands are frequently bright green to olive green. Rhinophores are lacking, labial tentacles are bow-shaped and tapered towards the ends (see Figures 1 and 2). Monaxone rodlet-like spicules distributed all over the body and frequently found in an accumulation between the oral tentacles are characteristic for Pontohedyle. These spicules can be confirmed for $P$. wenzli sp. nov., for P. yurihookeri sp. nov., P. milaschewitchii (Kowalevsky, 1901) and P. brasilensis (Rankin, 1979), and, in contrast to the original description [53], also in P. verrucosa. No spicules could be detected in $P$. peteryalli sp. nov. from Ghana. The absence of spicules is insufficient, however, to delineate microhedylid species, since their presence can vary under environmental influence [54]. 

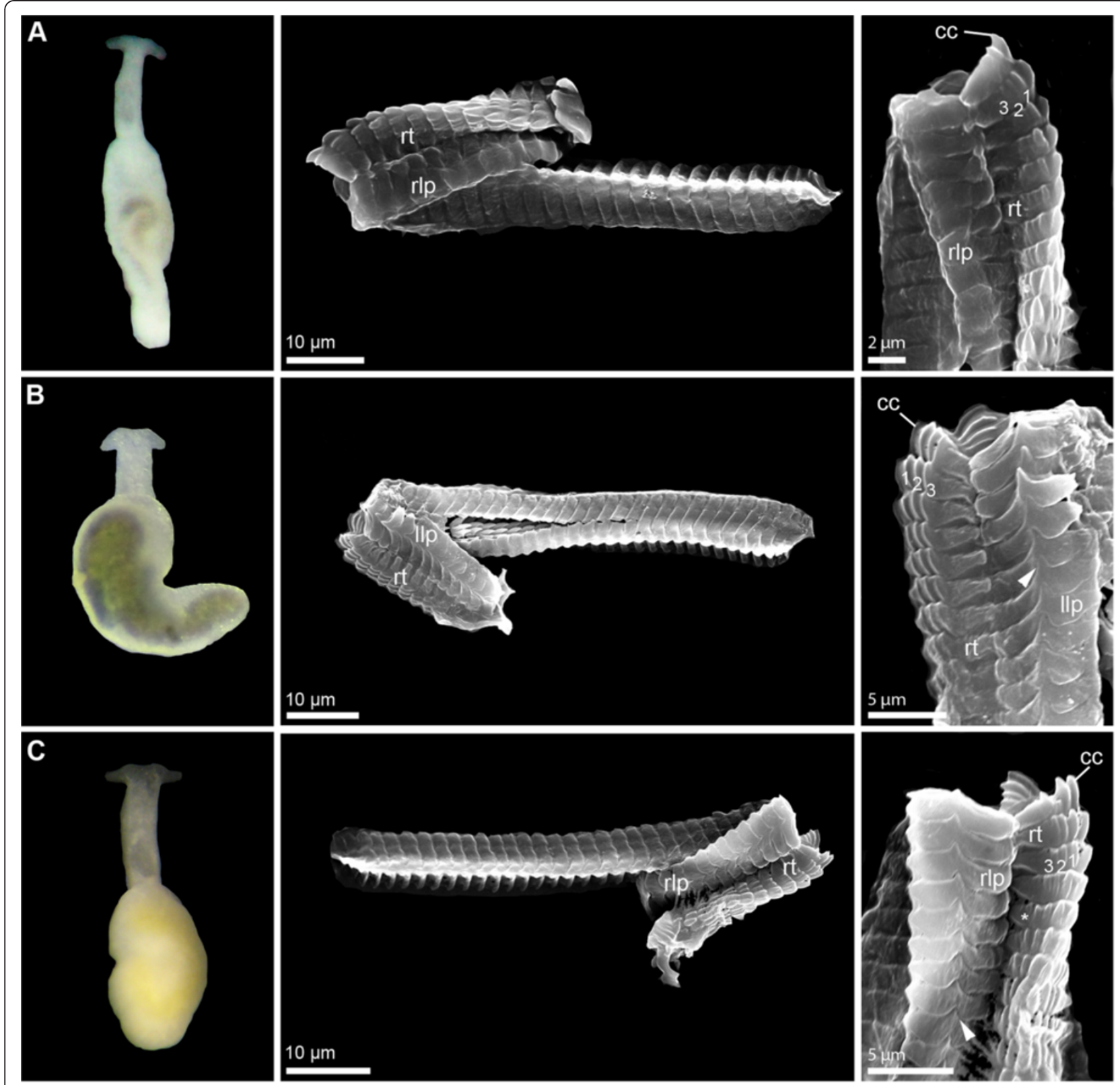

Figure 1 External morphology (living specimens) and radula characteristics (SEM micrographs) in Pontohedyle species (part 1).

A) Pontohedyle kepii sp. nov. (Pontohedyle sp. 1 in [25]); B) Pontohedyle joni sp. nov. (Pontohedyle sp. 2 from WA-5 (Belize) in [25]); C) Pontohedyle liliae sp. nov. (Pontohedyle sp. 4 in [25]), ${ }^{*}$ marks putative $4^{\text {th }}$ cusp on rhachidian tooth. $c c=$ central cusp of rhachidian tooth, IIp $=$ left lateral plate, $r$ lp $=$ right lateral plate, $\mathrm{rt}=$ rhachidian tooth.

The radulae of eight species were investigated using SEM (see Figures 1 and 2). Radulae of $P$. neridae sp. nov., $P$. martynovi sp. nov. and P. yurihookeri sp. nov. were not recovered whole from molecular preparations, and thus were unavailable for further examination [25]. The radula of $P$. wiggi sp. nov. could only be observed under the lightmicroscope, but not successfully transferred to a SEM stub. All radulae are hook-shaped with a longer dorsal and a shorter ventral ramus, typical for Acochlidia. Radula formulas are $38-53 \times 1.1 .1$, lateral plates are curved rectangular, and the rhachidian tooth is triangular and bears a central cusp and typically three smaller lateral denticles. Most radulae bear one pointed denticle centrally on the anterior margin of each lateral plate and a corresponding notch on the posterior side. Only the radula of $P$. kepii sp. nov. and P. verrucosa can be clearly distinguished from the others by the absence of this denticle and the more curved lateral teeth (see Figure 1A and [25], Figure 1D,E). Uniquely, $P$. verrucosa bears five lateral denticles next to the central cusp of the 

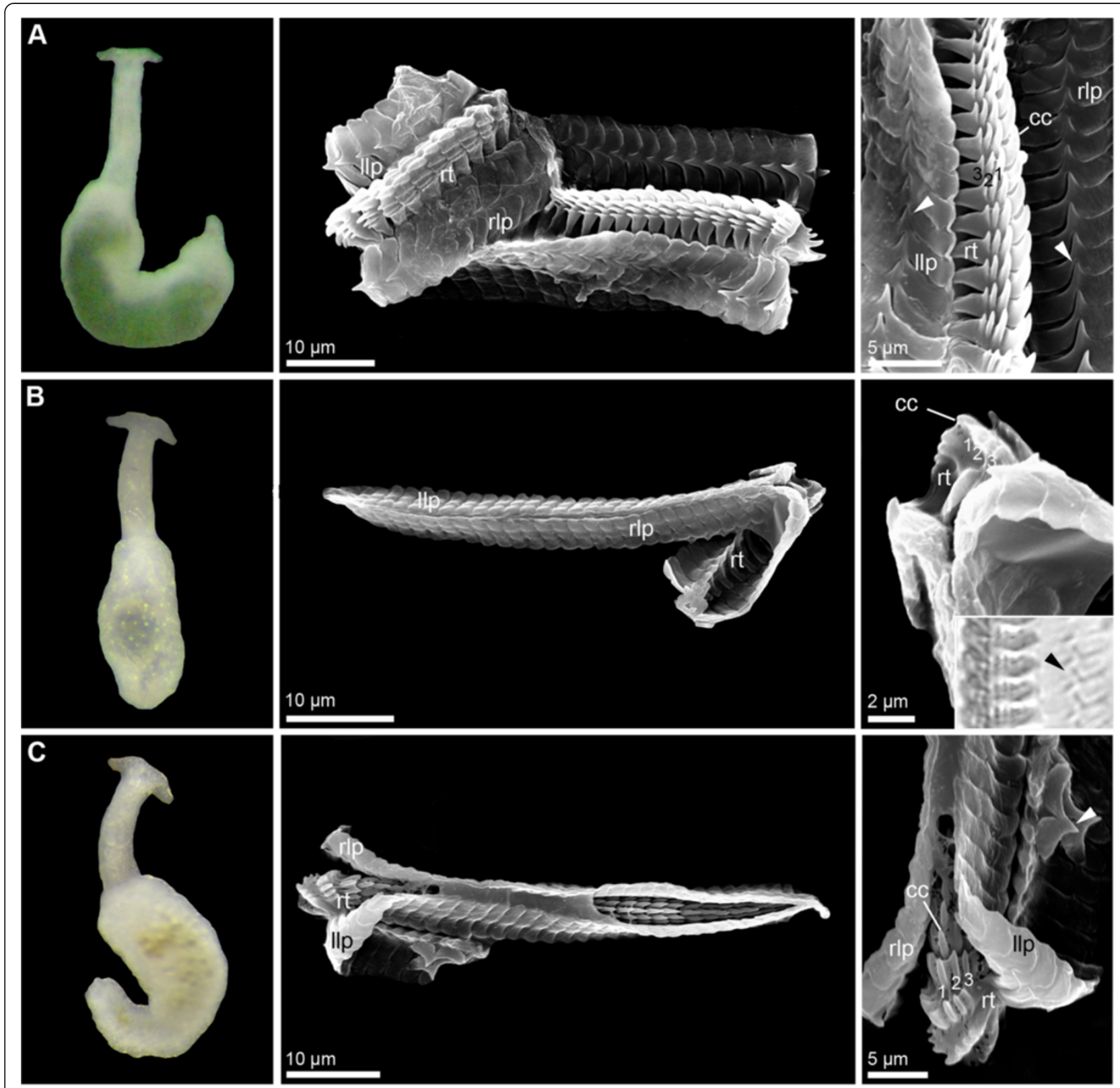

Figure 2 External morphology (living specimens) and radula characteristics (SEM micrographs) in Pontohedyle species (part 2).

A) Pontohedyle peteryalli sp. nov. (Pontohedyle sp. 7 in [25]); B) Pontohedyle wenzli sp. nov. (Pontohedyle sp. 6, picture of living animal from WP-1 (holotype), radula from IP-2, see [25]); C) P. brasilensis (living animal from WA-3 (Belize), radula from WA-10 (Brazil), see [25]). cc = central cusp of rhachidian tooth, $I \mathrm{p}=$ left lateral plate, $\mathrm{rlp}=$ right lateral plate, $\mathrm{rt}=$ rhachidian tooth.

rhachidian tooth [25]; in P. liliae sp. nov. a tiny fourth denticle borders the central cusp (see * in Figure 1C).

Previous phylogenetic analyses [25] recovered a deep split into two Pontohedyle clades: the P. milaschewitchii clade and the $P$. verrucosa clade. This is supported by novel analyses in a larger phylogenetic framework and additionally including a second nuclear marker (18S rRNA) (own unpublished data). Since no detailed histological account exists of any representative from the large $P$. verrucosa clade, we redescribe $P$. verrucosa (based on ZSM
Mol-20071833, 20071837 and 20100548), supplementing the original description with detailed information of the previously undescribed nervous and reproductive systems. The central nervous system (cns) of $P$. verrucosa lies prepharyngeal and shows an epiathroid condition. It consists of paired rhinophoral, cerebral, pleural, pedal and buccal ganglia and three unpaired ganglia on the visceral nerve cord, tentatively identified as left parietal ganglion, median fused visceral and subintestinal ganglion and right fused parietal and supraintestinal ganglion (Figure 3A). An 
osphradial ganglion or gastro-oesophagial ganglia were not detected. Anterior and lateral to the cerebral ganglia are masses of accessory ganglia. Due to the retracted condition of all examined specimens, tissues are highly condensed and no separation in different complexes of accessory ganglia could be detected. Attached to the pedal ganglia are large monostatolith statocysts. Oval, unpigmented globules are located in an antero-ventral position of the cerebral ganglia, interpreted as the remainder of eyes (see Figure 3B).

P. verrucosa is a gonochoristic species. The three sectioned specimens include two males and one female. The male reproductive system is comprised of gonad, ampulla, postampullary sperm duct, prostatic vas deferens, ciliated (non-glandular) vas deferens, genital opening and a small ciliated 'subepidermal' duct leading to a second genital opening anterodorsally of the mouth opening (Figure 3C). The sac-like gonad is relatively small and bears few irregular distributed spermatozoa. The large tubular ampulla emerges from the gonad without a detectable preampullary sperm duct; it is loosely filled with irregularly distributed spermatozoa (Figure 3D). The ampulla leads into a short, narrow ciliated postampullary duct widening into the large tubular prostatic vas deferens (staining pink in methylene-blue sections, Figure 3D). Close to the male genital opening, the duct loses its glandular appearance and bears cilia. The primary genital opening is located on the right side of the body at the visceral hump and close to the transition with the head-foot complex. Next to the genital opening, the anterior vas deferens splits off as an inconspicuous subepithelial ciliated duct that leads anteriorly on the right side of the head foot complex. It terminates in a second genital opening between the oral tentacles anterodorsally from the mouth opening.

The female reproductive system consists of gonad, nidamental glands and oviduct (Figure $3 \mathrm{E}$ ) and a genital opening located on the right side, in the posterior part of the visceral hump (not visible in Figure 3E, due to the retracted stage of the individual). The gonad is saclike and bears one large vitellogenic egg (see Figure 3F) and several developing oocytes. Three histologically differentiated tube-like nidamental glands could be detected with a supposedly continuous lumen and with an epithelium bearing cilia. From proximal to distal these glands are identified as albumen gland (cells filled with dark blue stained granules), membrane gland (pinkish, vacuolated secretory cells) and winding mucus gland (secretory cells stained pink-purple). In its proximal part the distal oviduct shows a similar histology as the mucous gland, but then loses its glandular appearance. The epithelium of the distal oviduct bears long, densely arranged cilia.

Additional notable histological features are numerous dark-blue-stained epidermal gland cells (see e.g., arrowhead in Figure 3D) and refracting fusiform structures in the digestive gland (see Figure 3B). An additional series of histological semi-thin sections of Pontohedyle kepii sp. nov. was sectioned and brief investigation revealed no variation in the major organization of the organ systems in Pontohedyle as described herein and in previous studies $[55,56]$.

\section{Remarks on the presentation of molecular characters}

Diagnostic characters for each species of Pontohedyle were extracted using the 'Characteristic Attribute Organization System' (CAOS) [51,57,58]. We define diagnostic characters as single pure characters, i.e. unique character states that respectively occur in all investigated specimens in a single Pontohedyle species but in none of the specimens of its congeners. As additional information single heterogeneous pure characters (i.e., different character states present within the species but absent from the congeners) are reported (for further details on the chosen approach see the Material and methods and Discussion sections). Positions refer to the position of the diagnostic nucleotide within the respective alignment (see Additional files 1, $2,3,4,5$ and 6). Where alignment positions differ from those in the deposited sequences, positions within the sequence of the holotype or in another reference sequence are also provided.

\section{Taxonomy of Pontohedyle}

Family: Microhedylidae Odhner, 1938 [59]

Genus: Pontohedyle Golikov \& Starobogatov, 1972 [60] Synonymy: Mancohedyle Rankin, 1979; Gastrohedyle

Rankin, 1979; Maraunibina Rankin, 1979

Type species (by subsequent designation): Pontohedyle milaschewitchii (Kowalevsky, 1901) [61]

Phylogenetic analyses of the genus Pontohedyle [25] confirmed earlier assumptions, that the three genera established by Rankin [62] (see above) present junior synonyms of Pontohedyle.

Morphological characteristics of genus Pontohedyle: Minute $(0.7-6 \mathrm{~mm})$ marine interstitial microhedylacean acochlid. Body divided into anterior head-foot complex and posterior visceral hump. In case of disturbance head-foot complex can be entirely retracted into visceral hump. Body whithish translucent. Foot with short rounded free posterior end. Head bears one pair of bow-shaped dorso-ventrally flattened oral tentacles. Rhinophores lacking. Monaxone, calcareous spicules irregularly distributed over head-foot complex and visceral hump. Radula hook-shaped band (lateral view), formula 1-1-1, lateral plates curved or with one pointed denticle, rhachidian tooth triangular with one central cusp and 2-4 lateral cusps on each side. Nervous 


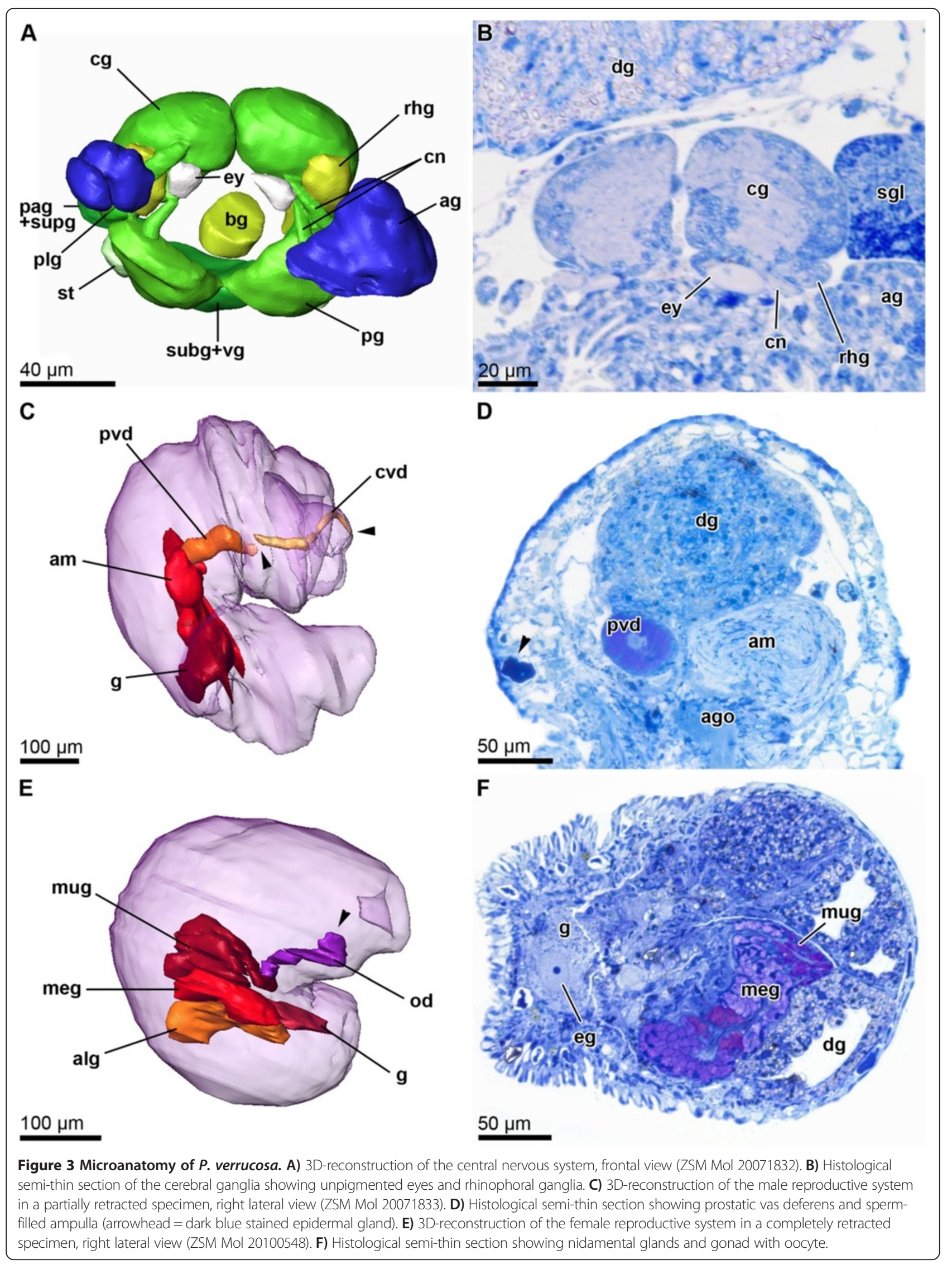


system with accessory ganglia at cerebral nerves anterior to the cns. Sexes separate, male reproductive system aphallic, sperm transferred via spermatophores.
Molecular diagnosis of the genus Pontohedyle, based on the sequences analyzed herein (Table 1) and on sequences from a set of outgroups including all acochlidian genera

Table 1 DNA sequence data analyzed in the present study to determine diagnostic nucleotides in Pontohedyle

\begin{tabular}{|c|c|c|c|c|c|c|}
\hline \multirow[t]{2}{*}{ Species } & \multirow[t]{2}{*}{ Museums number } & \multirow{2}{*}{$\begin{array}{l}\text { DNA } \\
\text { voucher }\end{array}$} & \multicolumn{4}{|c|}{ GenBank accession numbers } \\
\hline & & & $18 \mathrm{~S}$ rRNA & $28 \mathrm{~S}$ rRNA & 16S rRNA & COI \\
\hline \multirow[t]{5}{*}{ P. milaschewitchii } & ZSM Mol 20071381 & AB34404214 & - & JQ410926 & JQ410925 & JQ410897 \\
\hline & ZSM Mol 20080054 & AB34404241 & HQ168435 & $J F 828043$ & HQ168422 & - \\
\hline & ZSM Mol 20080055 & AB34404239 & - & - & JQ410927 & - \\
\hline & ZSM Mol 20080925 & - & - & - & JQ410928 & HQ168459 \\
\hline & ZSM Mol 20080953 & AB35081832 & KC984282 & - & JQ410929 & JQ410898 \\
\hline \multirow[t]{10}{*}{ P. brasilensis } & SI-CBC20 10KJ01-E03 & AB34500510 & KC984283 & JQ410941 & JQ410940 & - \\
\hline & SI-CBC20 10KJ01-B07 & AB34402082 & - & JQ410943 & JQ410942 & - \\
\hline & SI-CBC20 10KJ01-D07 & AB34500513 & - & JQ410944 & - & - \\
\hline & SI-CBC20 10KJ01-B09 & AB34402031 & - & JQ410946 & JQ410945 & JQ410904 \\
\hline & SI-CBC20 10KJ01-C09 & AB34500576 & - & JQ410948 & JQ410947 & JQ410905 \\
\hline & SI-CBC20 10KJ01-A10 & AB34402026 & - & - & JQ410949 & - \\
\hline & SI-CBC20 10KJ02-E01 & AB34402030 & - & JQ410950 & - & - \\
\hline & ZSM Mol 20110723 & AB34402034 & KC984284 & JQ410952 & JQ410951 & JQ410906 \\
\hline & ZSM Mol 20110722 & AB34402086 & KC984285 & JQ410932 & JQ410931 & JQ410900 \\
\hline & ZSM Mol 20090198 & AB35081813 & KC984286 & JQ410936 & JQ410935 & - \\
\hline \multirow[t]{7}{*}{$P$. verrucosa } & ZSM Mol 20071820 & AB34404223 & KC984287 & JQ410978 & JQ410977 & JQ410920 \\
\hline & ZSM Mol 20080176 & AB34404286 & - & JQ410980 & JQ410979 & JQ410921 \\
\hline & ZSM Mol 20071135 & AB34404221 & KC984288 & JQ410971 & JQ410970 & JQ410914 \\
\hline & ZSM Mol 20100388 & AB34500547 & - & - & - & JQ410916 \\
\hline & ZSM Mol 20100389 & AB34402044 & - & JQ410974 & - & JQ410917 \\
\hline & ZSM Mol 20100390 & AB34402070 & - & JQ410975 & - & JQ410918 \\
\hline & ZSM Mol 20100391 & AB34500531 & KC984289 & - & JQ410976 & JQ410919 \\
\hline Pontohedyle kepii sp. nov. & ZSM Mol 20081013 & AB35081769 & KC984290 & JQ410967 & JQ410966 & JQ410912 \\
\hline \multirow[t]{3}{*}{ Pontohedyle joni sp. nov. } & ZSM Mol 20090197 & AB34858164 & KC984291 & JQ410934 & JQ410933 & JQ410901 \\
\hline & SI-CBC20 10KJ01-D05 & AB34402049 & KC984292 & - & JQ410937 & JQ410902 \\
\hline & SI-CBC20 10KJ01-C08 & AB34402065 & - & JQ410939 & JQ410938 & JQ410903 \\
\hline Pontohedyle neridae sp.nov. & AM C. 476062.001 & AB34500497 & - & JQ410986 & JQ410985 & JQ410922 \\
\hline \multirow[t]{2}{*}{ Pontohedyle liliae sp.nov. } & ZSM Mol 20090471 & AB35081802 & KC984293 & JQ410954 & JQ410953 & - \\
\hline & ZSM Mol 20090472 & AB35081838 & - & JQ410956 & JQ410955 & - \\
\hline \multirow[t]{4}{*}{ Pontohedyle wiggi sp.nov. } & ZSM Mol 20100595 & AB34402059 & - & JQ410960 & JQ410959 & JQ410908 \\
\hline & ZSM Mol 20100596 & AB34402001 & - & - & JQ410961 & JQ410909 \\
\hline & ZSM Mol 20100597 & AB34500571 & - & JQ410963 & JQ410962 & JQ410910 \\
\hline & ZSM Mol 20100603 & AB34402020 & - & JQ410965 & JQ410964 & JQ410911 \\
\hline \multirow[t]{4}{*}{ Pontohedyle wenzli sp.nov. } & ZSM Mol 20100592 & AB34402021 & KC984294 & JQ410958 & JQ410957 & JQ410907 \\
\hline & AM C. 476051.001 & AB34402037 & KC984295 & JQ410982 & JQ410981 & - \\
\hline & ZSM Mol 20081014 & AB35081827 & KC984296 & JQ410969 & JQ410968 & JQ410913 \\
\hline & ZSM Mol 20100379 & AB34500521 & KC984297 & JQ410973 & JQ410972 & JQ410915 \\
\hline Pontohedyle peteryalli sp. nov. & ZSM Mol 20071133 & AB34404268 & KC984298 & - & JQ410930 & JQ410899 \\
\hline Pontohedyle martynovi sp. nov. & AM C. 476054.001 & AB34402062 & - & JQ410984 & JQ410983 & - \\
\hline Pontohedyle yurihookeri sp. nov. & ZSM Mol 20080565 & AB34402000 & KC984299 & JQ410987 & - & - \\
\hline
\end{tabular}


for which data are available $[63,64]$. Positions refer to the alignments in Additional files 1 and 2, and to the reference sequences of $P$. milaschewitchii, ZSM Mol 20080054 (GenBank HQ168435 and JF828043) from Croatia, Mediterranean Sea (confirmed to be conspecific with material collected at the type locality in molecular species delineation approaches [25]). Molecular diagnosis is given in Table 2.

Table 2 Molecular diagnostic characters of Pontohedyle

\begin{tabular}{ll}
\hline Marker & $\begin{array}{l}\text { Diagnostic characters with position } \\
\text { in alignment (in reference sequence) }\end{array}$ \\
\hline 18S rRNA & $165(168), G ; 1358(1365), A ; 1360$ \\
& $(1367), T ; 1371(1378), T ; 1514(1521), T$ \\
28S rRNA & $260, C ; 576, T ; 622, T$ \\
\hline
\end{tabular}

Pontohedyle milaschewitchii (Kowalevsky, 1901) [61]

Hedyle milaschewitchii Kowalevsky, 1901: p. 19-20 [61] Pontohedyle milaschewitchii (Kowalevsky) - Golikov \& Starobogatov [60]

Mancohedyle milaschewitchii (Kowalevsky) - Rankin (1979: p. 100) [62]

Pontohedyle milatchevitchi (Kowalevsky) - Vonnemann et al. (2005: p. 3) [65]; Göbbeler \& Klussmann-Kolb (2011: p. 122) [66].

Type locality: Black Sea, bay of St George monastery near Sevastopol, Crimean Peninsula, Ukraine.

Type material: To our knowledge no type material remains. Nevertheless we refrain from designating a neotype, as there is no taxonomic need, i.e. no possibility of confusion in the species' area of distribution.

Distribution and habitat: Reported from the Black Sea and numerous collecting sites throughout the Mediterranean e.g. $[55,61,67,68]$; marine, interstitial, subtidal 1-30 m, coarse sand.

Molecular diagnosis is given in Table 3.
ZSM Mol 20071381 (recollected at the type locality, see Figure 4) serves as the reference sequence, unless the sequence could not be successfully amplified. Then sequences (indicated below) from material from the Mediterranean serve as reference sequences (conspecifity was confirmed in a previous molecular species delineation approach 25]). Diagnostic characters in 18S rRNA were determined based on ZSM Mol 20080054 (GenBank HQ168435 = reference sequence) and ZSM Mol 20080953 (GenBank KC984282); in nuclear 28S rRNA based on ZSM Mol 20071381 (GenBank JQ410926) and ZSM Mol 20080054 (GenBank JF828043 = reference sequence), in mitochondrial 16S rRNA based on ZSM Mol 20071381 (GenBank JQ410925), ZSM Mol 20080054 (GenBank HQ168422), ZSM Mol 20080055 (GenBank JQ410927), ZSM Mol 20080925 (GenBank JQ410928) and ZSM Mol 20080953 (GenBank JQ410929), in mitochondrial COI based on ZSM Mol 20071381 (GenBank JQ410827), ZSM Mol 20080925 (GenBank HQ168459) and ZSM Mol 20080953 (GenBank JQ410898).

\section{Pontohedyle verrucosa (Challis, 1970) [53]}

Microhedyle verrucosa Challis, 1970: pp. 37-38 [53]

Pontohedyle verrucosa (Challis) - Wawra

(1987: p. 139) [69]

Maraunibina verrucosa (Challis) - Rankin (1979:

p. 102) [62]

Type locality: Coarse, clean shell sand, a little above low water at neap tide, near southern end of Maraunibina Island, Marau Sound, East Guadalcanal, Solomon Islands.

Type material: According to Challis [53] in the Natural History Museum, London, and the Dominion $\mathrm{Mu}-$ seum, Wellington, New Zealand. Own investigations revealed that the type material of Challis never arrived at the Natural History Museum, London and visiting the Museum of New Zealand Te Papa Tongarewa (former Dominion Museum), we were unable to locate any of her types. Thus, at current stage of

Table 3 Molecular diagnostic characters of Pontohedyle milaschewitchii

\begin{tabular}{|c|c|c|}
\hline Marker & $\begin{array}{l}\text { Diagnostic characters with position in } \\
\text { alignment (in reference sequence) }\end{array}$ & $\begin{array}{l}\text { Heterogeneous single } \\
\text { pure positions }\end{array}$ \\
\hline $18 \mathrm{~S}$ rRNA & 159, C; 164 (165), G & - \\
\hline $28 \mathrm{~S}$ rRNA & $329(324), T$ & - \\
\hline $16 \mathrm{~S}$ rRNA & $\begin{array}{l}\text { 8, G; 26, A; } 145 \text { (146), C; } 203 \text { (209), A; } 243 \text { (274), } \\
\text { G; } 275 \text { (306), T; } 290 \text { (321), T; } 333 \text { (363), A; } 352 \text { (382), T }\end{array}$ & 351 (381), T (G in ZSM Mol 20080953, position 381) \\
\hline $\mathrm{COI}$ & $\begin{array}{l}\text { 11, C; } 25, C ; 58, T ; 160, C ; 272, A ; 273, G ; 319, T ; 352, \\
\text { G; 371, G; 376, G; 397, A; 451, A; 476, C; 495, G; 496, G; 520, C }\end{array}$ & - \\
\hline $\mathrm{COI}(\mathrm{AA})$ & 4, L; 124, A; 159, L; 165, S & - \\
\hline
\end{tabular}




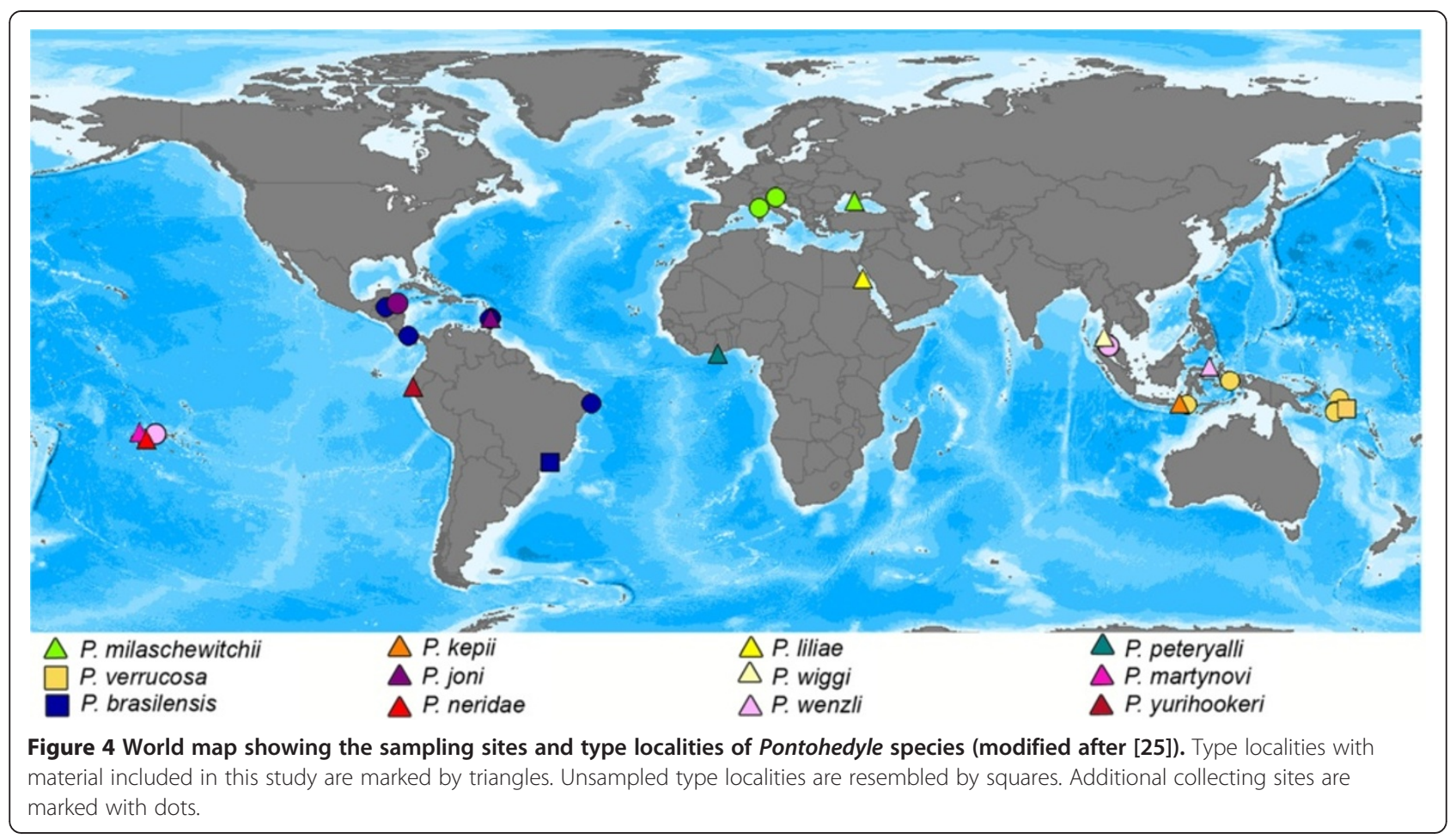

knowledge, type material might only remain in her private collection. We refrain from designating a neotype because we were unable to recollect at the type locality (see below).

Distribution and habitat: Reported from Indonesia and the Solomon Islands [25,53]; marine, interstitial, intertidal, coarse sand.

Sequenced material: In a collecting trip to the Solomon Islands, we were unfortunately unable to recollect at the type locality (Maraunibina Island, East Guadalcanal), but successfully recollected in Komimbo Bay (West Guadalcanal), a locality, from which the describing author noted similar ecological parameters and recorded several meiofaunal slug species occurring at both sites [53,70] Additional material was collected at different collecting sites in Indonesia (see Figure 4).

Molecular diagnosis is given in Table 4.
ZSM Mol 20071820 (from Komimbo Bay, East Guadalcanal, Solomon Islands) serves as the reference sequence. Diagnostic characters in nuclear $18 \mathrm{~S}$ rRNA were determined based on ZSM Mol 20071820 (GenBank KC984287), ZSM Mol 20071135 (GenBank KC984288) and ZSM Mol 20100391 (GenBank KC984289), in nuclear $28 \mathrm{~S}$ rRNA based on ZSM Mol 20071820 (GenBank JQ410978), ZSM Mol 20080176 (GenBank JQ410980), ZSM Mol 20071135 (GenBank JQ410971), ZSM Mol 20100389 (GenBank JQ410974) and ZSM Mol 20100390 (GenBank JQ410975), in mitochondrial 16S rRNA based on ZSM Mol 20071820 (GenBank JQ410977), ZSM Mol 20080176 (GenBank JQ410979), ZSM Mol 20071135 (GenBank JQ410970) and ZSM Mol 20100391 (GenBank JQ410976) and in mitochondrial COIbased on ZSM Mol 20071820 (GenBank JQ410920), ZSM Mol 20080176 (GenBank JQ410921), ZSM Mol 20071135 (GenBank JQ410914), ZSM Mol 20100388 (GenBank JQ410916),

Table 4 Molecular diagnostic characters of Pontohedyle verrucosa

\begin{tabular}{lll}
\hline Marker & $\begin{array}{l}\text { Diagnostic characters with position in } \\
\text { alignment (in reference sequence) }\end{array}$ & $\begin{array}{l}\text { Heterogeneous single } \\
\text { pure positions }\end{array}$ \\
\hline $18 \mathrm{~S}$ rRNA & - & - \\
$28 \mathrm{~S}$ rRNA & $597(605), \mathrm{T} ; 604(612), \mathrm{G}$ & - \\
$16 \mathrm{~S}$ rRNA & 235, deletion; $243(266), C ; 249(272), T ; 330(352), \mathrm{C}$ & - \\
COI & $118, \mathrm{~A} ; 343, \mathrm{G} ; 367, \mathrm{C} ; 421, \mathrm{~A} ; 451, \mathrm{C}$ & $541, \mathrm{~T}$ (C in ZSM 20080176, position 541) \\
\hline
\end{tabular}


ZSM Mol 20100389 (GenBank JQ410917), ZSM Mol 20100390 (GenBank JQ410918) and ZSM Mol 20100391 (GenBank JQ410919).

\section{Pontohedyle brasilensis (Rankin, 1979)}

Microhedyle milaschewitchii (Kowalevsky) - sensu Marcus (1953: pp. 219-220) [71]

Gastrohedyle brasilensis Rankin, 1979: p. 101 [62] Pontohedyle milaschewitchii (Kowalevsky) - sensu Jörger et al. (2007) [56], partim: all Western

Atlantic specimens.

Type locality: Shell gravel, intertidal, Vila, Ilhabela, São Paulo, Brazil.

Type material: No type material remaining in Marcus' collection (pers. comm. Luiz Simone). We nevertheless refrain from designating a neotype, since we lack material from the type locality.

Distribution and habitat: Caribbean Sea to southern Brazil [25,72]; marine, interstitial, intertidal to subtidal, coarse sand and shell gravel.

Sequenced material: Despite a series of recollecting attempts at the type locality and its vicinity in the past five years, we were unable to recollect any specimen of Pontohedyle in Southern Brazil. Our reference sequence refers to the southern-most specimen of a Western Atlantic Pontohedyle clade (see Figure 4), herein assigned to $P$. brasilensis (see Discussion). Additional material was collected at different collecting sites in the Caribbean (see Figure 4 for collecting sites and Figure $2 \mathrm{C}$ for photograph of a living specimen and SEM of radula).

Molecular diagnosis is given in Table 5.
Diagnostic characters in nuclear $18 \mathrm{~S}$ rRNA were determined based on ZSM Mol 20110722 from Pernambuco, Brazil (GenBank KC984285 = reference sequence), ZSM Mol 20110723 (GenBank KC984284), SI-CBC2010KJ01E03 (GenBank KC984283), ZSM Mol 20080198 (Gen Bank KC984286), in nuclear 28S rRNA based on ZSM Mol 20110722 (GenBank JQ410932); ZSM Mol 20090198 from St. Lucia Caribbean (GenBank JQ410936 = reference sequence); SI-CBC2010KJ01-E03 (GenBank JQ410941); SI-CBC2010KJ01-B07 (GenBank JQ410943), SI-CBC2010 KJ01-D07 (GenBank JQ410944); SI-CBC2010KJ01-B09 (GenBank JQ410946), SI-CBC2010KJ01-C09 (GenBank JQ410948), SI-CBC2010KJ02-E01(GenBank JQ410950), ZSM Mol 20110723 (GenBank JQ410952); in mitochondrial 16S rRNA based on ZSM Mol 20110722 (GenBank JQ410931 = reference sequence); ZSM Mol 20090198 (GenBank JQ410935); SI-CBC2010KJ01-E03 (GenBank JQ410940); SI-CBC2010KJ01-B07 (GenBank JQ410942), SI-CBC2010KJ01-B09 (GenBank JQ410945), SI-CBC2010 KJ01-C09 (GenBank JQ410947), SI-CBC2010KJ01-A10 (GenBank JQ410949), ZSM Mol 20110723 (GenBank JQ410951) and in mitochondrial COI based on ZSM Mol 20110722 (GenBank JQ410900= reference sequence); SI-CBC2010KJ01-B09 (GenBank JQ410904); SI-CBC2010KJ01-C09 (GenBank JQ410905); ZSM Mol 20110723 (GenBank JQ410906).

\section{Descriptions of new Pontohedyle species \\ Pontohedyle kepii sp. nov.}

Pontohedyle sp. 1 (MOTU I) in [25]

Types: Holotype: DNA voucher (extracted DNA in buffer, stored deep frozen at $-80^{\circ} \mathrm{C}$ ) ZSM Mol 20081013

Table 5 Molecular diagnostic characters of Pontohedyle brasilensis

\begin{tabular}{|c|c|c|}
\hline Marker & $\begin{array}{l}\text { Diagnostic characters with position in alignment } \\
\text { (in reference sequence) }\end{array}$ & Heterogeneous single pure positions \\
\hline $18 \mathrm{~S}$ rRNA & 164, T; 213 (225), G; 1693 (1706), T & - \\
\hline $28 \mathrm{~S}$ rRNA & $\begin{array}{l}648 \text { (654), A; } 653 \text { (659), T; 678, deletion, } 679 \\
\text { (684), T; } 683 \text { (688), T; } 704 \text { (709), C; } 801 \text { (806), T }\end{array}$ & $\begin{array}{l}564 \text { (570), T (in SI-CBC2010KJ01-B09 and ZSM 20090198: } \\
\text { A); } 793 \text { (798) , C (in SI-CBC2010KJ02-E01: T, position 682) }\end{array}$ \\
\hline $16 \mathrm{~S}$ rRNA & $\begin{array}{l}\text { 1, T; 11, deletion; } 18 \text { (17), A ; } 80 \text { (81), T; } 102 \text { (103), G; } 107 \\
\text { (108), T; 131, G; 142, C; } 172 \text { (173), C; } 182 \text { (184), A; } 210 \text { (212), } \\
\text { A; 214, deletion; } 288 \text { (306), G; } 308 \text { (325), C; } 359 \text { (376), C; } 369 \text { (386), G }\end{array}$ & - \\
\hline $\mathrm{COl}$ & 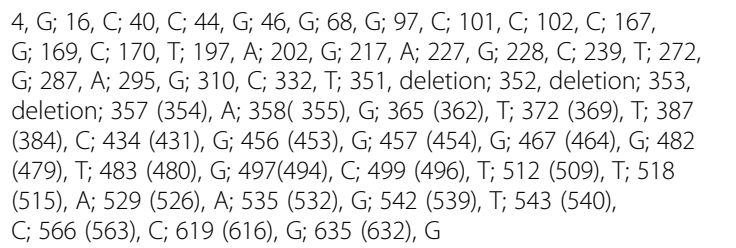 & $\begin{array}{l}\text { 70, A (in ZSM Mol 20110722, G); 205, T (in ZSM } \\
\text { Mol 20110722, C); 517, T (in ZSM Mol 20110722, C); }\end{array}$ \\
\hline $\mathrm{COI}(\mathrm{AA})$ & $\begin{array}{l}\text { 4, I; 15, A; 23, V; 32, T; 34, P; 56, V; 57, L; 66, I; 76, A; 80, L; 91, A; 96, } \\
\text { M; 111, L; 118, E; 119, deletion; } 124 \text { (123), F; } 129 \text { (128), A; } 145 \text { (144), } \\
\text { V; } 152(151), W ; 156(155), A ; 161 \text { (160), W; } 171 \text { (170), L; } 173 \text { (172), I; } \\
176 \text { (175), L; } 189 \text { (188), L; } 212 \text { (211), V }\end{array}$ & - \\
\hline
\end{tabular}


(DNA bank accession number AB35081769). Paratypes: two specimens fixed in $96 \%$ ethanol were lost during DNA extraction. Two specimens fixed in glutaraldehyde and embedded in epoxy resin (ZSM 20080877 and 20080977). ZSM 20080877 sectioned at $1 \mu \mathrm{m}$. One additional specimen dissolved for radula preparation, SEM stub with radula available (ZSM Mol 20131101). All material collected at type locality.

Type locality: S $8^{\circ} 13^{\prime} 59^{\prime \prime}$, E $117^{\circ} 28^{\prime} 32^{\prime \prime}$; Pulau Moyo, Nusa Tengarra, Indonesia, Flores Sea, Indo Pacific (see Figure 4).

ZooBank registration: urn:lsid:zoobank.org:act:694022 A2-BE21-4082-8CFD-A66094740A95

Etymology: Named after our good friend and longtime diving companion, Klaus-Peter ('Kepi') Schaaf, who assisted us in collecting sand samples during diving in Indonesia.

Distribution and habitat: Currently known from type locality only; marine, interstitial, subtidal 5-6 m, coarse coral sand.

Description: morphologically with diagnostic characters of the genus Pontohedyle (see Figure 1A). Radula formula 1-1-1, rhachidian tooth with three lateral cusps, lateral plate smooth without denticle (Figure 1A).

Molecular diagnosis is given in Table 6.

Table 6 Molecular diagnostic characters of Pontohedyle kepii sp. nov.

\begin{tabular}{|c|c|}
\hline Marker & $\begin{array}{l}\text { Diagnostic characters with position in } \\
\text { alignment (in reference sequence) }\end{array}$ \\
\hline $18 \mathrm{~S}$ rRNA & $\begin{array}{l}199 \text { (182), G; } 202 \text { (185), C; 203, deletion; 204, deletion; } \\
\text { 206, deletion; } 254 \text { (244), T; } 707 \text { (697), T; } 1355 \\
\text { (1345), A; } 1356 \text { (1346), C }\end{array}$ \\
\hline $28 \mathrm{~S}$ rRNA & 410 (439), T; 419 (448), C; 719 (754), G; 867 (902), C \\
\hline 16S rRNA & $\begin{array}{l}\text { 11, T; } 184 \text { (189), A; } 187 \text { (192), C; } 239 \text { (267), A; 242, } \\
\text { deletion; 243, deletion; 244, deletion; } 294 \\
\text { (324), G; } 302 \text { (328), G }\end{array}$ \\
\hline $\mathrm{COI}$ & $\begin{array}{l}49, \mathrm{~A} ; 79, \mathrm{~T} ; 118, \mathrm{C} ; 148, \mathrm{C} ; 160, \mathrm{~A} ; 193, \mathrm{G} ; 292, \mathrm{G} ; \\
331, \mathrm{G} ; 466, \mathrm{~T} ; 494, \mathrm{G} ; 583, \mathrm{G} ; 628, \mathrm{~A} ; 638, \mathrm{C}\end{array}$ \\
\hline $\mathrm{COI}(\mathrm{AA})$ & $165, \mathrm{D}$ \\
\hline
\end{tabular}

Positions of the diagnostic characters refer to the sequence of the holotype. Diagnostic characters in nuclear $18 \mathrm{~S}$ rRNA were determined based on GenBank KC984290, in 28S rRNA based on GenBank JQ410967, in mitochondrial 16S rRNA based on GenBank JQ410966, and in mitochondrial COI based on GenBank JQ410912.

\section{Pontohedyle joni sp. nov.}

\section{Pontohedyle sp. 2 (MOTU II) in [25]}

Types: Holotype: DNA voucher (extracted DNA in buffer) ZSM Mol 20090197 (DNA bank accession number AB34858164). Paratype: one specimen fixed in $96 \%$ ethanol, collected with the holotype.

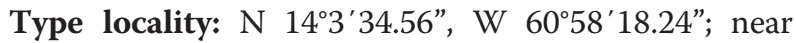
Castries, St. Lucia, Central America, Caribbean Sea, West Atlantic Ocean (see Figure 4).

Additional material: DNA voucher (extracted DNA in buffer) SI-CBC2010KJ01-D05 (DNAbank at ZSM AB34402049) and SEM preparation of radula (ZSM Mol 20131102) from $N 16^{\circ} 48^{\prime} 13.44^{\prime \prime}$, W 88 $44^{\prime} 36.9^{\prime \prime}$, and DNA voucher (extracted DNA in buffer) SICBC2010KJ01-C08 (DNAbank AB34402065) from $\mathrm{N}$ $16^{\circ} 48^{\prime} 7.62^{\prime \prime}$, W 88 $44^{\prime} 36.42^{\prime \prime}$ both Carrie Bow Cay, Belize, Central America, Caribbean Sea, West Atlantic Ocean.

ZooBank registration: urn:lsid:zoobank.org:act:73AA C79D-5A43-40E4-B0D6-0329CAAA2AA0

Etymology: Named after Dr. Jon Norenburg to honor his efforts and enthusiasm for meiofaunal research and to thank him for his support for uncovering the largely unknown Caribbean meiofauna.

Distribution and habitat: Currently known from the Caribbean Sea (St. Vincent and Belize), type locality subtidal, 2-3 $\mathrm{m}$ depth, sand patches between seagrass, coarse sand. Additional material also subtidal, 14-15 m, sand patches between corals, coarse sand.

Description: morphologically with diagnostic characters of the genus Pontohedyle. Radula formula $48 \times 1-1-1$, rhachidian tooth with 3 lateral cusps, lateral plate with one pointed denticle (see Figure 1B).

Molecular diagnosis is given in Table 7.

Table 7 Molecular diagnostic characters of Pontohedyle joni sp. nov.

\begin{tabular}{|c|c|c|}
\hline Marker & Diagnostic characters with position in alignment (in reference sequence) & Heterogeneous single pure positions \\
\hline $18 \mathrm{~S}$ rRNA & 207 (215), T; 209 (217), T; 256 (263), A & - \\
\hline $28 \mathrm{~S}$ rRNA & 443 (446), A; 547 (556), T; 868 (873), A & \\
\hline $16 \mathrm{~S}$ rRNA & $\begin{array}{l}44 \text { (47), C; } 122 \text { (125), T; } 141 \text { (144), A; } 142 \text { (145), G; } 143 \text { (146), G; 146, G; } 152 \text { (157), } \\
\text { A; } 182 \text { (188), T; } 236 \text { (252), A; } 259 \text { (284), C }\end{array}$ & $\begin{array}{l}181 \text { (187), } \mathrm{T} \text { (in SI-CBC20 10KJ01-C08, } \\
\mathrm{C} \text { at position 187) }\end{array}$ \\
\hline $\mathrm{COI}$ & 31, A; 85, G; 160, G; 283, G; 298, G; 451, G; 523, C; 526, A; 578, C; 580, T & \\
\hline
\end{tabular}


The sequences retrieved from the holotype ZSM Mol 20090197 serve as reference sequences. Diagnostic characters in nuclear 18S rRNA were determined based on ZSM Mol 20090197 (GenBank KC984291) and SICBC2010KJ01-D05 (GenBank KC984292), in nuclear 28S rRNAbased on ZSM Mol 20090197 (GenBank JQ410934) and SI-CBC2010KJ01-C08 (GenBank JQ410939), in mitochondrial 16S rRNA based on ZSM Mol 20090197 (GenBank JQ410933), SI-CBC2010KJ01-D05 (GenBank JQ410937) and SI-CBC2010KJ01-C08 (GenBank JQ41 0938), and in mitochondrial COI based on ZSM Mol 20090197 (GenBank JQ410901), SI-CBC2010KJ01-D05 (GenBank JQ410902) and SI-CBC2010KJ01-C08 (GenBank JQ410903).

\section{Pontohedyle neridae sp. nov.}

\section{Pontohedyle sp. 3 (MOTU III) in [25]}

Types: Holotype: DNA voucher (extracted DNA in buffer, stored deep frozen at $-80^{\circ} \mathrm{C}$ ) AM C. 476062.001 (DNA bank accession number at ZSM AB34500497). Paratype: one specimen fixed in 5\% formalin and embedded in epoxy resin (AM C.476063.001), collected with the holotype.

Type locality: S 17³2'50.172", W 14946'35.4"; Motu Iti, Moorea, Oceania, Central Pacific Ocean (see Figure 4).

ZooBank registration: urn:lsid:zoobank.org:act:BE3E 7920-5451-429D-95E4-C8D2F859C7CB

Etymology: Named after our friend and colleague, Dr. Nerida Wilson, with a big 'thank you' for actively sharing with us the fascination for interstitial Acochlidia.

Distribution and habitat: Known from type locality only; subtidal 3-4 m, fine to medium coral sand.

Description: Morphologically with diagnostic characters of the genus Pontohedyle. Radula characteristics unknown.

Molecular diagnosis is given in Table 8.

Table 8 Molecular diagnostic characters of Pontohedyle neridae sp. nov.

\begin{tabular}{ll}
\hline Marker & $\begin{array}{l}\text { Diagnostic characters with position } \\
\text { in alignment (in reference sequence) }\end{array}$ \\
\hline $28 \mathrm{~S}$ rRNA & $61(57), \mathrm{G} ; 522(518), \mathrm{A}$ \\
16S rRNA & $11, \mathrm{G} ; 121(123), \mathrm{T} ; 145(147), \mathrm{T} ; 147$ \\
& $(149), \mathrm{G} ; 252(276), \mathrm{C} ; 263(286), \mathrm{T} ; 330$ \\
& $(352), \mathrm{G} ; 336(358), \mathrm{G}$ \\
$\mathrm{COI}$ & $46, \mathrm{C} ; 151, \mathrm{C} ; 169, \mathrm{G} ; 220, \mathrm{~A} ; 277, \mathrm{C} ;$ \\
& $278, \mathrm{~T} ; 289, \mathrm{~T} ; 391, \mathrm{C} ; 397, \mathrm{G} ; 421, \mathrm{C} ;$ \\
& $479, \mathrm{~T} ; 505, \mathrm{~A} ; 601, \mathrm{C}$ \\
\hline
\end{tabular}

The sequences retrieved from the holotype serve as reference sequences. Diagnostic characters in nuclear 28S rRNA were determined based onAM C. 476062.001 (GenBank JQ410986), in mitochondrial 16S rRNA based on AM C. 476062.001 (GenBank JQ410985), and in mitochondrial COI based on AM C. 476062.001 (GenBank JQ410922).

\section{Pontohedyle liliae sp. nov.}

\section{Pontohedyle sp. 4 (MOTU IV) in [25]}

Types: Holotype: DNA voucher (extracted DNA in buffer, stored deep frozen at $-80^{\circ} \mathrm{C}$ ) ZSM Mol 20090471 (DNA bank accession number AB35081802). Paratypes (all collected with the holotype): DNA voucher (extracted DNA in buffer) ZSM Mol 20090472 (DNA bank accession number AB35081838), one additional specimen used for radula preparation, SEM stub with radula available (ZSM Mol 20131103).

Type locality: N 2411'50“, E 35³8'26" (approximation from Google Earth), Sha'ab Malahi, Egypt, Africa, Red Sea (see Figure 4).

ZooBank registration: urn:Isid:zoobank.org:act:2711E 3E5-1D1D-41B0-B919-7D7E690FD525

Etymology: Named after Reinhilde ('Lili') Schmid, our friend and diving companion, who assisted us during sand collecting in Egypt and shares our fascination for this world of little creatures.

Distribution and habitat: Known from type locality only; subtidal $20 \mathrm{~m}$, relatively fine coral sand.

Description: Morphologically with diagnostic characters of the genus Pontohedyle. Radula formula $45 \times 1-1-1$, rhachidian tooth with three (to four) lateral cusps, lateral plate with one pointed denticle (Figure 1C). Eyes clearly visibly externally, monaxone spicules in accumulation between oral tentacles and irregular all over the body.

Molecular diagnosis is given in Table 9.

\begin{tabular}{|c|c|}
\hline Marker & $\begin{array}{l}\text { Diagnostic characters with position in alignment } \\
\text { (in reference sequence) }\end{array}$ \\
\hline $18 \mathrm{~S}$ rRNA & $\begin{array}{l}\text { 33, C; 40, C; 54, G; 117, T; 129, T; } 146 \text { (147), C; } 149 \text { (150), T; } \\
186 \text { (187), C; } 214 \text { (223), A; } 215 \text { (224), C; } 623 \text { (631), T; } 663 \text { (673), } \\
\text { T; } 677 \text { (687), C; } 841 \text { (853), G; } 959 \text { (971), G; } 1028 \text { (1040), T; } \\
1030 \text { (1042), C; } 1348 \text { (1360), A; } 1363 \text { (1375), T }\end{array}$ \\
\hline $28 \mathrm{~S}$ rRNA & $\begin{array}{l}34 \text { (30), C; } 63 \text { (59), C; } 536 \text { (532), T; } 537 \text { (533), G; 542, deletion; } \\
555 \text { (554), G; } 590 \text { (589), T; } 642 \text { (641), C; } 643 \text { (642), T; } 658 \text { (657), } \\
\text { A; } 671 \text { (670), C; } 696 \text { (695), A; 827, G; 837, C; } 902 \text { (904), C }\end{array}$ \\
\hline $16 \mathrm{~S}$ rRNA & $\begin{array}{l}\text { 10, C; } 211 \text { (222), C; } 246 \text { (277), C; } 330 \text { (359), T; } 336 \text { (365), C; } \\
357 \text { (386), C }\end{array}$ \\
\hline
\end{tabular}


The sequences retrieved from the holotype (ZSM Mol 20100471) serve as reference sequences. Diagnostic characters in nuclear $18 \mathrm{~S}$ rRNA were determined based on ZSM Mol 20100471 (GenBank KC984293), in nuclear 28S rRNA based on ZSM Mol 20100471 (GenBank JQ410954) and ZSM Mol 20100472 (GenBank JQ410956), and in mitochondrial 16S rRNA based on ZSM Mol 20100471 (GenBank JQ410953) and ZSM Mol 20100472 (GenBank JQ410955).

\section{Pontohedyle wiggi sp. nov.}

Pontohedyle sp. 5 (MOTU V) in [25]

Types: Holotype: DNA voucher (extracted DNA in buffer) ZSM Mol-20100595 (DNA bank accession number AB34402059). Paratypes (all collected with the holotype): DNA voucher (extracted DNA in buffer) ZSM Mol20100596 (DNA bank AB34402001), ZSM Mol 20100597 (DNA bank AB34500571), ZSM Mol 20100603 (DNA bank AB34402020); one specimen fixed in glutaraldehyde and embedded in epoxy resin (ZSM Mol 20100598).

Type locality: N 7³6'15“, E 98²2'37“, Ko Raccha Yai, Phuket, Thailand, Andaman Sea, Indian Ocean (see Figure 4).

ZooBank registration: urn:lsid:zoobank.org:act:808E5 62E-0E1A-4D79-BB2C-1377B3734F86

Etymology: Named in memory of Ludwig ('Wigg') Demharter, a malacologist friend, passionate diver, 'fun researcher', and for many years a supporter of the ZSM and the second author's working group.

Distribution and habitat: Known from the type locality only; marine, interstitial between sand grains, relatively fine coral sand, subtidal 6-7 $\mathrm{m}$ depth, sandy slope among patches of corals.

Description: Morphologically with diagnostic characters of the genus Pontohedyle. Radula formula 1-1-1, lateral plate with one pointed denticle (as in P. milas chewitchii). Eyes visibly externally, monaxone spicules present.

Molecular diagnosis is given in Table 10.

Table 10 Molecular diagnostic characters of Pontohedyle wiggi sp. nov.

\begin{tabular}{ll}
\hline Marker & $\begin{array}{l}\text { Diagnostic characters with position } \\
\text { in alignment (in reference sequence) }\end{array}$ \\
\hline 28 S rRNA & $\begin{array}{l}483(472), T ; 508(497), T ; 536, \text { deletion; 537, } \\
\text { deletion; 538, deletion; } 699(687), A\end{array}$ \\
$16 S$ rRNA & $180(188), C ; 374(406), T$ \\
COI & $127, C ; 325, A ; 583, C$ \\
COI (AA) & $29, T$ \\
\hline
\end{tabular}

The sequences retrieved from the holotype (ZSM Mol 20090595) serve as reference sequences. Diagnostic characters in nuclear 28S rRNA were determined based on ZSM Mol 20100595 (GenBank: JQ410960), ZSM Mol 20100597 (GenBank: JQ410963), ZSM Mol 20100603 (GenBank: JQ410965), in mitochondrial 16S rRNA based on ZSM Mol 20100595 (GenBank: JQ410959), ZSM Mol 20100596 (GenBank: JQ410961), ZSM Mol 20100597 (GenBank: JQ410962), ZSM Mol 20100603 (GenBank: JQ410964), and in mitochondrial COI based on ZSM Mol 20100595 (GenBank: JQ410908), ZSM Mol 20100596 (GenBank: JQ410909), ZSM Mol 20100597 (GenBank: JQ410910), ZSM Mol 20100603 (GenBank: JQ410911).

\section{Pontohedyle wenzli sp. nov.}

\section{Pontohedyle sp. 6 (MOTU VIII) in [25]}

Types: Holotype: DNA voucher (extracted DNA in buffer) ZSM Mol 20100379 (DNA bank accession number AB34500521).

Type locality: N $1^{\circ} 27^{\prime} 53^{\prime \prime}$, E $125^{\circ} 13^{\prime} 48^{\prime \prime}$, Lembeh Strait, Sulawesi, Indonesia, Banda Sea, West Pacific Ocean (see Figure 4).

Additional material DNA voucher (extracted DNA in buffer) ZSM Mol 20081014 (DNA bank accession number AB35081827) and one specimen used for SEM preparation of radula (available at ZSM Mol 20131105), locality S 8²3'58“, E $119^{\circ} 18^{\prime} 56^{\prime \prime}$, Pulau Banta, Nusa Tengarra, Indonesia Flores Sea, Indo-Pacific. DNA voucher (extracted DNA in buffer) ZSM 20100592 (DNA bank AB34402021), locality N 7³6'15“, E 98 22'37“, Ko Raccha Yai, Phuket, Thailand, Andaman Sea, Indian Ocean. DNA voucher (extracted DNA in buffer) AM C. 476051.001 (DNA bank AB34402037) and one specimen fixed in $5 \%$ formalin and embedded in epoxy resin (AM C.476050.001), locality S 17²8'33.96", W 14949'51.6”, E of Cook's Bay Pass, Moorea, Oceania, Central Pacific.

Note: Most species delineation approaches suggested ZSM 20100592, and some also AM C. 476051.001, as an independently evolving lineage [25]. Due to the conservative consensus approach, these specimens were included in the described species. Future analyses might show that their separation as independent species is warranted.

ZooBank registration: urn:lsid:zoobank.org:act:558E C548-1FB3-4B00-B248-4424CA7B098C

Etymology: Named after Alexander Wenzl, for his support during the development of this manuscript and his interest for meiofaunal research.

Distribution and habitat: Known from Indonesia, with putative distribution across the Indo-Pacific and Central Pacific; marine, subtidal (3-22 m), interstitial, coarse sand and shell grid. 
Description: Morphologically with diagnostic characters of the genus Pontohedyle, eyes clearly visible externally (see Figure 2B, picture of living holotype). Radula $43 \times 1-1-1$, rhachidian tooth with three lateral cusps, lateral plate with pointed denticle (like in P. milaschewitchii).

Molecular diagnosis is given in Table 11.
Additional material: six specimens in 75\% Ethanol collected at Nzema Cape, Ghana, Africa, Gulf of Guinea, East Atlantic Ocean; conspecifity still needs to be confirmed via barcoding.

ZooBank registration: urn:lsid:zoobank.org:act:B25E5 0F7-F0D2-4842-B6C3-5A79EA784A0C

Table 11 Molecular diagnostic characters of Pontohedyle wenzli sp. nov.

\begin{tabular}{lll}
\hline Marker & Diagnostic characters with position in alignment (in reference sequence) & Heterogeneous single pure positions \\
\hline 18S rRNA & $771(791), T ; 772(792), T$ & - \\
28S rRNA & $449(455), C ; 539(545), A$ & - \\
16S rRNA & $36, G ; 41, T ; 84(88), A ; 143(147), A ; 144(148), A ; 161(167), T ; 176(182), A ; 194$ & $332(370), A(Z S M$ Mol 20081014, \\
& $(201), T ; 207(214), A ; 256(296), C ; 258(298), A ; 269(309), T ; 295$, deletion; 331 & G at position 370) \\
& $(369), A ; 340(378), A$ & - \\
COI & $181, A ; 218, G ; 219, T ; 296, T ; 383, C ; 430, T ; 593, A$ & - \\
COI (AA) & $73, V ; 94, F ; 122, A ; 198, I$ & \\
\hline
\end{tabular}

The sequences retrieved from the holotype (ZSM Mol 20100379) serve as reference sequences. Diagnostic characters in nuclear $18 \mathrm{~S}$ rRNA were determined based on ZSM Mol 20100379 (GenBank KC984297), ZSM Mol 20081014 (GenBank KC984296), ZSM Mol 20100592 (GenBank KC984294), AM C. 476051.001 (GenBank KC984295), in nuclear $28 S$ rRNA based on ZSM Mol 20100379 (GenBank JQ410973), ZSM Mol 20081014 (GenBank JQ410969), ZSM Mol 20100592 (GenBank JQ410958), AM C. 476051.001 (GenBank JQ410982), in mitochondrial 16S rRNA based ZSM Mol 20100379 (GenBank JQ410972), ZSM Mol 20081014 (GenBank JQ410968), ZSM Mol 20100592 (GenBank JQ410957), AM C. 476051.001 (GenBank JQ410981), and in mitochondrial COI based on ZSM Mol 20100379 (GenBank JQ410915), ZSM Mol 20081014 (GenBank JQ410913), ZSM Mol 20100592 (GenBank JQ410907).

\section{Pontohedyle peteryalli sp. nov.}

\section{Pontohedyle sp. 7 (MOTU VII) in [25]}

Types: Holotype: DNA voucher (extracted DNA in buffer) ZSM Mol 20071133 (DNA bank accession number AB34404268). Paratypes (all collected with the holotype): eight specimens preserved in 96\% ethanol (ZSM Mol 20070827); four in 75\% ethanol (ZSM Mol 20070827), sixteen specimens fixed in glutaraldehyde, post-fixed in osmium and embedded in epoxy resin (ZSM Mol 20080453-60; ZSM Mol 20080462-69). SEM stub with radula available (ZSM Mol 20131104).

Type locality: N $04^{\circ} 47^{\prime} 46^{\prime \prime}$, W $02^{\circ} 10^{\prime} 06^{\prime \prime}$, MiaMia, Ghana, Africa, Gulf of Guinea, East Atlantic Ocean (see Figure 4).
Etymology: Named for our friend and malacologist, Peter ('Pete') Ryall, who invited us to explore sea slugs right in front of his MiaMia home.

Distribution and habitat: Currently only known from the Ghana West Coast around MiaMia, marine, interstitial, subtidal 2-3 m, fine sand.

Description: Morphologically with diagnostic characters of the genus Pontohedyle. Radula $42 \times 1-1-1$, rhachidian tooth with three lateral cusps, lateral plate with pointed denticle (like in $P$. milaschewitchii), see Figure 2A.

Molecular diagnosis is given in Table 12.

\begin{tabular}{|c|c|}
\hline Marker & $\begin{array}{l}\text { Diagnostic characters with position in } \\
\text { alignment (in reference sequence) }\end{array}$ \\
\hline $18 \mathrm{~S}$ rRNA & $160, C_{;} 164, C$ \\
\hline $\mathrm{COI}$ & $\begin{array}{l}14, T ; 23, A ; 48, C ; 68, A ; 76, C ; 81, T ; 83, A ; 95, T ; 101, A ; \\
102, G ; 140, A ; 141, C ; 167, A ; 187, C ; 209, C ; 232, C ; 280, \\
\text { A; 286, C; 293, A; 294, G; 357, C; 358, A; 361, A; 365, A; 373, } \\
\text { A; 433, C; 448, G; 467, A; 468, T; 487, T; 503, T; 504, G; 512, } \\
\text { A; 535, C; 556, C; 574, A; 586, C; 628, C; 634, C }\end{array}$ \\
\hline $\mathrm{COI}(\mathrm{AA})$ & $\begin{array}{l}\text { 5, L; 8, I; } 16, \mathrm{~A} ; 23, \mathrm{I} ; 27, \mathrm{~V} ; 28, \mathrm{~T} ; 32, \mathrm{~S} ; 34, \mathrm{~S} ; 47, \mathrm{~T} ; 56, \mathrm{I} ; 70, \\
\mathrm{~L} ; 119, \mathrm{~T} ; 156, \mathrm{I} ; 162, \mathrm{D} ; 168, \mathrm{C} ; 171, \mathrm{I}\end{array}$ \\
\hline
\end{tabular}

The sequences retrieved from the holotype (ZSM Mol 20071133) serve as reference sequences. Diagnostic characters in nuclear $18 \mathrm{~S}$ rRNA were determined based on GenBank KC984298, in mitochondrial 16S rRNA based GenBank JQ410930 and in mitochondrial COI based on GenBank JQ410899. 
Pontohedyle martynovi sp. nov.

\section{Pontohedyle sp. 8 (MOTU IX) in [25]}

Types: Holotype: DNA voucher (extracted DNA in buffer) AM C. 476054.001 (DNA bank accession number at ZSM AB34402062). Paratype: one specimen fixed in 5\% formalin embedded in epoxy resin (AM C.476053.001), collected together with the holotype.

Type locality: S $17^{\circ} 28^{\prime} 17^{\prime \prime}$, W $149^{\circ} 48^{\prime} 42^{\prime \prime}$, E of Cook's Bay Pass, Moorea, Oceania, Central Pacific Ocean (see Figure 4).

ZooBank registration: urn:lsid:zoobank.org:act:9431E 4B8-EAF3-4E29-9993-BCD7C52928C6

Etymology: Named to thank our Russian friend and taxonomist, Alexander ('Sasha') Martynov, for collecting acochlidians for us in many places, including Pontohe dyle milaschewitchii at its type locality.

Distribution and habitat: Known from type locality only; marine, interstitial, subtidal 18-20 m, coarse sand, shell grid and rubble.

Description: Morphologically with diagnostic characters of the genus Pontohedyle. Radula characteristics unknown.

Molecular diagnosis is given in Table 13.

Table 13 Molecular diagnostic characters of Pontohedyle martynovi sp. nov.

\begin{tabular}{ll}
\hline Marker & $\begin{array}{l}\text { Diagnostic characters with position } \\
\text { in alignment (in reference sequence) }\end{array}$ \\
\hline 28S rRNA & $539(541), C ; 623(629), A$ \\
16S rRNA & 8, deletion; $33(32), T ; 130(131), C ; 144$, deletion; 151 \\
& $(155), G ; 168(172), G ; 171(175), A ; 218(232), A ; 230, T ;$ \\
& 232 (244), G; 235 (258), C; 242 (274), C; 332 (365), \\
& C; $334(367), G ; 353(386), G ; 373(408), G$ \\
\hline
\end{tabular}

The sequences retrieved from the holotype (AM C. 476054.001) serve as reference sequences. Diagnostic characters in nuclear $28 \mathrm{~S}$ rRNA were determined based on GenBank JQ410984, and in mitochondrial 16S rRNA based on GenBank JQ410983.

\section{Pontohedyle yurihookeri sp. nov.}

\section{Pontohedyle sp. 9 (MOTU X) in [25]}

Types: Holotype: DNA voucher (extracted DNA in buffer) ZSM Mol 20080565 (DNA bank accession number AB34402000).

Type locality: S 358'55”, W 80 59'10", Punta Sal, Peru, South America, East Pacific Ocean (see Figure 4).

ZooBank registration: urn:Isid:zoobank.org:act:9B858 AA5-59FA-4505-AE94-FB2EA27FBEF6
Etymology: Named for our Peruvian friend and marine biologist, Yuri Hooker, who joined us during a great diving expedition to explore the Peruvian sea slug fauna.

Distribution and habitat: Known from type locality only; marine, interstitial, subtidal ( $8 \mathrm{~m})$, coarse sand.

Description: Morphologically with diagnostic characters of the genus Pontohedyle. Radula characteristics unkown.

Molecular diagnosis is given in Table 14.

Table 14 Molecular diagnostic characters of Pontohedyle yurihookeri sp. nov.

\begin{tabular}{ll}
\hline Marker & $\begin{array}{l}\text { Diagnostic characters with position } \\
\text { in alignment (in reference sequence) }\end{array}$ \\
\hline 18S rRNA & $163(156), T ; 200(193), A ; 213$ (225), A; 770 (783), \\
& T; $810(823), T$ \\
28S rRNA & $110(139), A ; 398(427), T ; 399(428), T ; 403$ (432), T; 409 (438), \\
& A; 410, deletion; 413 (441), G; 436 (464), T; 445, deletion; 446, \\
& deletion; 447 (473), C; 449 (475), A; 451 (477), A; 452 (478), \\
& A; 457 (483), A; 460 (486), T; 477 (503), C; $563(593), T$ \\
\hline
\end{tabular}

The sequences retrieved from the holotype (ZSM Mol 20080565) serve as reference sequences. Diagnostic characters in nuclear $18 \mathrm{~S}$ rRNA were determined based on GenBank KC984299, and in nuclear 28S rRNA based on GenBank JQ410987.

\section{Discussion}

\section{Cryptic species challenging traditional taxonomy}

Largely due to the development of molecular methods, research on cryptic species has increased over the past two decades $[8,9]$, demonstrating their commonness across Metazoan taxa, though with random or non-random distribution among taxa and biomes still to be investigated $[9,10]$. Several recent studies have underlined that there is a large deficit in alpha taxonomy and that the diversity of marine invertebrates and especially meiofaunal animals might be much higher than expected, partly caused by high proportions of cryptic species e.g., $[11,13,14,25,73-75]$. Rather than global, amphi-Oceanic, circum-tropical or otherwise wide ranging, the distribution areas of the biological meiofaunal species involved may be regional and their ecology more specialized $[12,25,76]$. At an initial stage of molecular and ecological exploration, cryptic meiofauna is potentially threatened by global change and cannot effectively be included in conservation approaches.

In traditional taxonomy, most species descriptions are based on morphological and anatomical characters. Morphological species delineation, however, can fail to 
adequately address the diversity of life on Earth by leaving cryptic species unrevealed. Many taxonomists agree that the future of taxonomic descriptions should be integrative, embracing all available data sources (morphology, molecular sequences, biogeography, behavioral traits...) that can contribute to species delineation [1-3]. Previous authors have argued that 'integrative taxonomy' does not necessarily call for a maximum of different character sets, but rather requires the taxonomist to select character sets adequate for species delineation in the particular group of taxa [3,5]. Thus, there should be no obligation in taxonomic practice to stick to morphology as the primary source [77], and there are no official requirements by the International Code of Zoological Nomenclature to do so $[78,79]$.

The results of Jörger et al. [25] indicate that the members of Pontohedyle slug lineages are so extremely uniform that conventional taxonomic characters (i.e. external morphology, radula characteristics, spicules) fail to delineate species. A series of studies have demonstrated the generally high potential of advanced 3D-microanatomy for character mining in Acochlidia (e.g., [80-82]). However, the exclusively mesopsammic microhedylacean Acochlidia form an exception, as they show reduced complexity in all organ systems and uniformity that leaves few anatomical features for species delineation even on higher taxonomic levels [83]. Based on previous histological comparisons, Jörger et al. [56] were unable to find any morphological characters justifying discrimination between the closely related western Atlantic P. brasilensis and its Mediterranean congener, $P$. milaschewitchii. Here, we provided a detailed histological (re-)description using 3D-reconstruction based on serial semi-thin sections of $P$. verrucosa, to evaluate whether advanced 3D-microanatomy provides distinguishing morphological characters for the two generally accepted species, $P$. milaschewitchii and $P$. verrucosa, as representatives of the two major Pon tohedyle clades (see [25], Figure 1). Indeed, we revealed some putative distinguishing features in the reproductive and digestive systems (see Table 15). However, the encountered (minor) morphological differences are problematic to evaluate in the absence of data on ontogenetic and intraspecific variation, and on potential overlap with interspecific differences. For example, slight differences in the reproductive system could be due to different ontogenetic stages, therefore presently they cannot be used to discriminate species. Comparatively investigated serial semi-thin sections of Pontohedyle kepii sp. nov. also confirmed the similarity in all major organ systems reported previously $[55,56]$. We thus conclude that in Pontohedyle even advanced microanatomy is inefficient or even inadequate for species diagnoses. Molecular character sets currently offer the only chances for unambiguous discrimination between the different evolutionary lineages. Proponents of morphology based alpha taxonomy [84] might argue that we have not attempted a fully integrative approach since we have not performed 3D-microanatomy on all proposed new species, including enough material for intra-specific comparisons, ultrastructural data on, e.g., cilia, sperm morphology or specific gland types, to reveal whether these forms indeed represent cryptic species. However, in light of the biodiversity crisis and the corresponding challenges to taxonomy, we consider it as little effective to dedicate several years of a taxonomist's life to the search for morphological characters, when there is little to expect, while molecular characters enable straightforward species delineation. This is not a plea to speed up description processes at the expense of accuracy and quality, or by allowing ignorance of morphology, but for a change in taxonomic practice to give molecular characters similar weight as morphological ones, in cases in which this is more informative or practical.

Still debated is the way how the traditional Linnaean System needs to be adapted to incorporate different character sets, in the first place the growing amount of molecular data. Probably the most radical way ignores the character-based requirements of the International Code of Zoological Nomenclature [78,79] and proposes to base descriptions of new species directly on support

Table 15 Putative distinguishing features between $P$. milaschewitchii and $P$. verrucosa (intraspecific variation not evaluated)

\begin{tabular}{lll}
\hline & P. milaschewitchii (Kowalevsky, 1901) & P. verrucosa (Challis, 1970) \\
\hline Data source & Jörger et al. $2008[55]$ & Present study \\
Epidermal glands & Predominantly whitish, blue stained only in & Predominantly whitish and numerous dark blue \\
& one small row & stained ones \\
Nervous system & Eyes pigmented and externally visible & Eyes unpigmented \\
Reproductive system & Only one cephalic male genital opening detected & Two male genital openings (cephalic and visceral) \\
$\begin{array}{l}\text { Digestive system/ putatively } \\
\text { different feeding habits }\end{array}$ & Lateral radula teeth with central denticle & Lateral radula teeth without denticle \\
& Lipid-like droplets in digestive gland & Refracting fusiform structures \\
\hline
\end{tabular}


values under species delineation models $[85,86]$. Aside from the paradigm shift this would bring, far away from long-standing taxonomic practice, opponents criticize that unambiguous allocation of newly collected material is impossible in the absence of definitions and descriptors and requires repetition of the species delineation approach applied [50]. As a method of species delineation, coalescent based approaches are objective and grounded on evolutionary history and population genetics [86,87]; thus it is indeed tempting to use results derived from molecular species delineations approaches directly as species descriptions ('model-based species descriptions' [87]). This would clearly facilitate descriptions, thus reduce the taxonomic impediment and the risk of an endless number of discovered but undescribed candidate species. Every species description should aim for differentiation from previously described species; therefore, diagnostic characters are usually derived from comparisons to other, closely related species. Nevertheless, the species description itself has to be self-explanatory and should not rely on comparative measurements which are only valid in comparison to a special set of other species used for a certain analysis, i.e. on a complex construct that may not be reproducible when new data are added. In contrast to Fujita \& Leaché [87], we believe that each species, i.e. separately evolving lineage [4], will present - in the current snap-shot of evolutionary processes - fixed diagnostic characters of some sort (e.g., from morphology, DNA sequence information, behavioral, karyology...), and we consider it the task of modern taxonomy to detect the most reliable and efficient set of characters on which to found species descriptions.

The Characteristic Attribute Organization System (CAOS) $[51,57,58]$ is a character based method proposed for uniting species discovery and description [88]. As an approach to species delineation, we consider it inferior to coalescent based approaches (e.g., GMYC and BP\&P); CAOS successfully determines putative diagnostic nucleotides, but is not predictive, i.e. lacks objective criteria with which to delimit a threshold number of distinguishing nucleotides that would indicate a species boundary. One has to distinguish between diagnosability of entities and the delimitation of species. Diagnostic characters of whatever sort can be found for all levels in the hierarchical classification, but there is no objective criterion for determining a number of characters needed to characterize a (new) species, e.g. versus a population. Nevertheless, for the purpose of species description, we think that character based approaches like CAOS are highly valuable and should complement molecular species delineation procedures, thus enabling the transition from species discovery to description.

\section{Requirements of molecular taxonomy}

While calls for replacing the Linnaean system by a DNA sequence based one [41] have trailed away, we still lack a common procedure on how to include molecular data into the Linnaean system [21]. Like any other source of data, molecular data is not explicitly treated by the International Code of Zoological Nomenclature, there are no provisions dictating the choice of characters [78,79]. Currently, molecular data are included in species descriptions in various mutually inconsistent ways [21]. If DNA sequence data are only used as additive to, e.g., morphology based species descriptions or molecular species delineation approaches to confirm pre-identified entities, the addition is straightforward and requires no specific considerations. But if molecular sequence information is to be used as the partial or even sole content of a species description, a discussion of the corresponding best practice is needed.

\section{Type material for species based on molecular data}

Previous authors highlighted the need for voucher material in molecular studies [89]. Ideally, DNA is extracted from (a subsample of) a name-bearing type specimen (holotype, syntype, lectotype or neotype); if no such specimen is available for molecular studies, an attempt should be made to collect fresh material at the type locality. If parts of larger animals belonging to putative new species are used for DNA extraction, DNA and remaining specimen can both become part of the type material under nomenclatural rules. However, where the members of a putatively new species, e.g. of meiofauna, are so small that molecular extraction from only part of an individual is impossible, taxonomists may be confronted with the critical decision to either have DNA without a morphological type specimen or a type without DNA. In taxonomically unproblematic groups one can add new material or use paratypes for DNA (or other) analyses, relying on specimens to be conspecific if they were collected from 'the same population', i.e. from a place (and time) close enough to the type locality to assume gene flow. But what if, as has been shown for Pontohedyle slugs [25], there is a possibility of cryptic species occurring sympatrically and at the same time? Would it be better (A) to sacrifice a (single available) type specimen to obtain molecular data for species delineation or (B) to save the type and use a secondary specimen, taking the risk that the latter might not be conspecific with the former? In a group like our Pontohedyle slugs in which DNA sequence data are much more promising for species delineation than morphological approaches, and considering the wealth of potential DNA sequence characters, we prefer to sacrifice even single specimens to DNA extraction. In absence of a term referring to vouchers exclusively consisting of extracted DNA, we term this type material: 'DNA types'. However, prior to this, researchers should attempt an optimization of microscopical documentation (for details see [90]) and recovery of hard parts (e.g. 
radulae) from the spin columns used for extraction [91]. In the case of DNA aliquots serving as type material, natural history collections are urged to create long term DNA storage facilities $[41,42]$ like the DNA bank network (http:// www.dnabank-network.org/), and should apply the same caution and requirements (i.e. documentation of collection details) as for any morphological type.

\section{Risk of two parallel taxonomies?}

Old type material often does not allow molecular analyses $[84,92]$, and searching for fresh material at a type locality can be unsuccessful. Future technical advances are likely to enable DNA acquisition from some old type material, as there has been considerable progress in dealing with degenerated DNA [93]. Nevertheless, there are the potential risks that two parallel taxonomic systems could develop, and that the one based on molecular characters could duplicate, under separate names, some taxa already established on morphological grounds [77]. Similar concerns have arisen previously when the taxonomy of certain taxa was based on a character set other than morphology (e.g. cytotaxonomy based on data from chromosomes) and the investigation of one character set hindered the exploration of the other. It clearly remains the duty of taxonomists to carefully check type material of closely related taxa before describing new species [77]. To keep molecule driven taxonomy 'workable' [94] and connected to traditional morphology based taxonomy, authors should include a brief morphological diagnosis of the (cryptic) species [77], even in the absence of species-diagnostic characters, in order to make the species recognizable as belonging to a certain group of (cryptic) species.

\section{Trouble with names}

Any specimen identified from molecular data only can belong to a previously established species or to one new to science. If unambiguous identification with a single existing species name is possible then, of course, the latter should be used. In our cases in Pontohedyle, we call those Indo-Pacific specimens collected near the type locality of P. verrucosa (Challis, 1970) on the Solomon Islands by this single available name for Indo-Pacific Pontohedyle. Concerning Atlantic Pontohedyle, the name $P$. brasilensis (Rankin, 1979), proposed for Brazilian specimens, was treated as a junior synonym of the older name, P. milaschewitschii (Kowalevsky, 1901). Since we have shown that $P$. milaschewitschii refers to Mediterranean and Black Sea specimens only [25], we resurrected the name P. brasilensis for Western Atlantic Pontohedyle, and now apply it to the only species in of two cryptic ones that has been collected from Brazil. In doing so we accept the risk resulting from the fact that these specimens were collected at some distance from the type locality of P. brasilensis (see Figure 4), as the latter has not yielded any Pontohedyle specimens for more than the last 50 years, despite considerable and repeated collecting efforts, including our own. These assignments of previously established species names left at least nine additional, clearly separate Pontohedyle species for which available names did not exist. In cases of microscopic animals such as Pontohedyle, molecular taxonomy thus may benefit from morphology based taxonomy having missed them in the past.

\section{Species descriptions based on singletons}

Species descriptions based on singleton specimens cannot reflect intraspecific variation, and Dayrat [1] even proposed a guideline to restrict species descriptions to well-sampled taxa. However, there is no objective way to determine any sample size at which intraspecific variation would be covered sufficiently. Moreover, excluding taxa described from singletons would lead to considerably lower, and effectively false, estimates of the scientifically known biodiversity [5,26-28]. The present study on Pontohedyle includes five species descriptions based on DNA sequence information from one individual only. Usually, this is done when such a singleton presents a combination of characters so discrete that it is considered highly unlikely to fall within the variational range of another species [28]. In a complex molecular species delineation approach Jörger et al. [25] recognized our five singletons as independently evolving lineages. Approximations with molecular clock analyses estimate the diversification of these species from their respective sister groups to have occurred 54-83 mya (own unpublished data), which indicates significant timespans of genetic isolation. In light of our general revision of the genus Pontohedyle, we consider it as less productive to keep these entities on the formally unrecognized level of candidate species than to run the risk that our species hypotheses may have to be modified due to future additional material. Nevertheless, we are well aware of the fact that taxon sampling and data acquisition (i.e. incomplete molecular data sets) are not yet ideal for some of our newly described species (e.g., P.martynovi sp. nov., P. yurihookeri sp. nov.).

\section{What is a diagnostic character in molecular taxonomy?}

In character based taxonomy, descriptions of new taxa are, or should be, based on diagnostic differences from previously known taxa. In a phenetic framework (key systematics), similarity based distinction relies on sufficient sampling and detectable degrees of difference, whereas phylogenetic taxonomy additionally presumes knowledge of character homologies and sister group relationships. In an ideal phylogenetic framework diagnoses are based on apomorphic (i.e. derived) versus homologous but plesiomorphic (ancestral) states of a given character. In 
molecular taxonomy, the detection of homologies and apomorphic conditions among the four character states (bases) is handicapped by the high chance of convergent multiple transformations causing homoplasy. Reconstruction of ancestral sequences to support homology and differentiate between apomorphic and plesiomorphic character states for each node is possible [95]. However, unfortunately, robust phylogenetic hypotheses with strong support values for all sister group relationships are the exception rather than the rule. Since the evaluation of a state as apomorphic highly depends on the topology, and reconstruction of ancestral nucleotides is constrained by sampling coverage, we suggest more conservative approaches for cases of unclear phylogenetic relationships, as in our study. We use diagnostic nucleotides as unique character attributes (which may be apomorphic or plesiomorphic or convergent) within a certain entity, i.e. a monophylum with strong support values. This is clearly a trade off between the number and phylogenetic significance of diagnostic characters and the degree of dependence of these characters on a certain topology, as with increasing size and diversity of the selected entity, the likelihood of homoplasy also rises [96]. To enhance the stability of our molecular taxonomic characters we chose to determine diagnostic characters of each Pontohedyle species in relation to all its congeners, rather than just to the respective sister taxon as is the default in CAOS. Equal character states in non-Pontohedyle outgroups are left unconsidered, however, due to the larger evolutionary distances and the correspondingly increased risk of homoplasies. It will be one of the major challenges for molecule driven taxonomy to select the appropriate monophylum in which all included taxa are evaluated against each other. Rach et al. [88] addressed homoplasy within the selected ingroup by applying an $80 \%$ rule to so-called single private characters (see below). Pontohedyle species recognized here offered enough single pure diagnostic bases to avoid using single private characters and some further, more equivocal attributes provided by CAOS.

The Characteristic Attribute Organization System (CAOS) $[51,57,58]$ can be used to identify diagnostic nucleotides for pre-defined taxonomic units [51]. The program offers discrimination between four types of 'character attributes' (CAs): simple (single nucleotide position) vs. compound (set of character states) and pure vs. private [51]. Pure CAs are nucleotides present in all members of a clade and absent from members of other clades; private CAs are only present in some members of the clade, but absent from others [51]. We consider only single pure CAs as eligible for diagnostic characters in DNA taxonomy, i.e. as supporting new species proposals. In our diagnoses of the new Pontohedyle species we emphasize those single pure CAs, which in protein coding genes code for a different amino acid. The probability of single pure CAs referring to fixed genetic differences increases exponentially with their number [88]. In our dataset, all Pontohedyle species have between 12 and 36 single pure CAs on independently evolving markers, which supports their treatment as genetically isolated lineages. Additionally, the CAOS program distinguishes between homogeneous pure CAs (shared by all members of the taxon under study, and not present in the outgroups) and heterogeneous pure CAs (with two or three different characters present in the taxon but absent from the outgroups). The latter characters can be treated as diagnostic, but are problematic as they may refer to convergently evolved character states. Therefore, we report them as additional information. In contrast, compound CAs can be unique for certain species, but they may have evolved from several independent mutation events. Consequently, compound CAs as an entity have low probabilities of homology; in analogy to morphoanatomical key systematics, these compound CAs can serve for re-identification of well-sampled species, but they are not diagnostic characters in a phylogenetic sense and thus should be avoided in DNA taxonomy.

CAOS identifies discrete nucleotide substitutions at every node of a given tree and has been complemented to find diagnostic bases in a 'phylogenetic-free context' [97], referring to the difference between CAs and true apomorphies. This notion can be misleading, however, as the results provided by CAOS are one hundred percent topology dependent in only comparing sister pairs at each node. To overcome this topology dependence, we ran several analyses placing each species at the root of the ingroup, which we defined as the most inclusive secure and taxonomically relevant monophylum, in our case the genus Pontohedyle (see Material and Methods). This procedure of a manually iterative, exhaustive intrageneric comparison of base conditions makes the recognized single pure CAs less numerous but more rigorous than with CAOS default parameters, i.e. by decreasing the chances of homoplasy and increasing the chances of single pure CAs representing apomorphies in our wider taxon comparison.

\section{Towards a 'best practice' in molecular taxonomy}

Considering stability and traceability in future research, the presentation of the identified diagnostic nucleotides is not trivial. Some recent studies just reported the number of differing nucleotides without specifying the position and character state e.g., [98]. This is equivalent to a morphological species description that would merely refer to, e.g., 'diagnostic differences in the reproductive system' without offering any descriptive details. Other studies present part of an alignment without identifying positions, and underline putative diagnostic nucleotides 
e.g., [99] without explanation what determined these bases as diagnostic. This practice leaves it to future researchers to identify the proposed bases, which is highly time consuming and error-prone, especially when the original alignment is not deposited in a public database. Reporting the positions within the alignment is a step towards reproducibility and traceability of molecular diagnostic characters e.g., [94,100-102], but when new material is added that was generated with different primers or includes insertions or deletions, the critical positions are still difficult to trace. Yassin et al. [103] included the positions within a reference genome, which probably provides the greatest clarity for future research. Unfortunately, for non-model taxa closely related reference genomes which allow for unambiguous alignment of even fast evolving markers are usually unavailable. We thus suggest the following procedure for reporting positions in an alignment. (1) Clearly report primers and alignment programs, and clarify what determined position 1 (e.g., first base after the primer sequence); (2) deposit alignments in public databases or as additional material accompanying the publication's online edition. To make a diagnostic position in a sequence traceable independently from a specific alignment, we additionally recommend to (3) report the corresponding position in a deposited reference sequence (ideally generated from type material). Technically, the necessary values are easily retrievable from sequence editing programs such as Geneious [104]. To evaluate intraspecific variation, sequences from all specimens assigned to a certain species were included in our analyses of diagnostic characters. In new species descriptions the provided reference sequences should be generated from type material. In cases where the molecular data retrieved from the type are, however, incomplete, we consider it little problematic to additionally include data from other specimens, if there is justification on conspecifity (e.g. via other molecular markers). If future research rejects conspecifity, the respective characters can be easily excluded from the original description. We refrain from adopting the term 'genetype', however, as label for sequences data from type material [105], as it might be easily misunderstood: sequences themselves are not types but amplified copies of certain parts of type material.

Since an alignment presents the positional homology assumptions that are crucial for the determination of diagnostic nucleotides, we consider the quality of the alignment as essential for the success of molecular taxonomy. Therefore, we sincerely recommend to critically compare the output of different alignment programs, as in the present study. While coding mitochondrial markers (such as COI) can be checked via reading frames and translation into amino acids, and are generally less problematic, non-coding fast evolving markers (e.g. $16 \mathrm{~S}$
rRNA) can be difficult to align even among closely related species. Obviously, undetected misalignments can result in tremendous overestimation of diagnostic characters. For example, a misalignment occurred in the ClustalW approach to our $28 \mathrm{~S}$ rRNA dataset, which increased the number of characters diagnostic for a sister clade within Pontohedyle wenzli sp. nov. on this marker from 0 to 34 compared to the MUSCLE [106] alignment. And even without obvious misalignments, the use of different alignment programs can result in a differing number of diagnostic nucleotides (e.g. 9 vs. 13 diagnostic nucleotides in $P$. milaschewitchii comparing the MUSCLE and ClustalW alignment). By removing ambiguous parts of the alignment, one reduces the number of diagnostic characters considerably (e.g. from 19 to 13 diagnostic nucleotides on $16 \mathrm{~S}$ rRNA in $P$. milaschewitchii when masking ClustalW alignments with Gblocks [107]). However, those diagnostic characters that remain can be considered as more stable and reliable for species identification. Based on our comparative analyses, we decided to choose the most conservative approach (alignment conducted with MUSCLE [106] and masked with GBlocks [107]), and based on the above mentioned examples stress the need to dedicate time to alignment issues when performing molecular taxonomy.

Several potential sources of error unique to taxonomy from molecular data have been pointed out [23]. (1) contamination and chimeric sequences, (2) faulty alignments resulting in comparisons of non-homologous nucleotides, and (3) the risk of dealing with paralogs. Authors of species descriptions based on molecular data should bear these pitfalls in mind. The risk of chimeric sequences can be reduced by carefully conducting BLAST searches [108] for each amplified fragment; misidentifications of diagnostic characters due to non-homologous alignments can be avoided by applying the considerations discussed above. The quality and stability of molecular taxonomic results considerably increase when several independent loci support the species delineation. To avoid idiosyncrasies of individual markers, misidentifications due to sequencing errors, or the pitfalls of paralogs, we strongly recommend not to base molecular species delineation and subsequent species description on single markers. Otherwise, if subsequent results negate the diagnostic value of nucleotides on that marker, the species description loses its entire foundation. Furthermore, the use of single pure CAs rather than of other types of CAs, and especially the use of genus-level compared CAs as discussed above, increases the chances of establishing and diagnosing new species on apomorphies rather than on homoplasies.

We acknowledge the risk that species descriptions based on molecular data might contain errors in the form of incorrectly assumed apomorphies, especially when working in sparsely sampled groups. Moreover, 
putative molecular apomorphies of described species may have to be reconsidered as plesiomorphies when new species with the same characteristics are added, or they may vanish in intraspecific variation. The more potentially apomorphic nucleotides are found across independently evolving markers, the higher the chances that at least some of them truly refer to unique mutations accumulated due to the absence of gene exchange. But in all this, molecular characters do not differ from morphological or other sets of characters. Species descriptions are complex hypotheses on several levels: novelty of taxon, placement within systematic context, and hypothesis of homology applying descriptive terms $[5,109,110]$. Species descriptions based on molecular characters are founded on the well-established hypothesis that character differences reflect lineage independence [50] and that mutations accumulate in the absence of gene exchange. It is the task of the taxonomist to evaluate whether the observed differences in character states can be explained by a historical process causing lineage divergence [3]. According to rough time estimations by molecular clock analyses, the radiation of Pontohedyle species included in the present study took place 100-25 mya (own unpublished data). Therefore we are confident that many of the bases recognized as diagnostic within our sampling truly refer to evolutionary novelties and unique attributes of species-level entities. However, even in cases of more recent divergences it should be possible to detect at least some diagnostic bases. Regardless of which character set a species description is based on, species descriptions are hypotheses, which means that they need to be re-evaluated, i.e. confirmed, falsified or modified when new data, material or methods of analysis become available.

\section{Conclusions}

This contribution issues a plea to follow up discoveries of cryptic species by molecular species delineation with the steps necessary to establish formal scientific names for these species. This can be achieved by selection of diagnostic characters, e.g., via the CAOS software. Depending on the robustness of the underlying phylogenetic hypothesis, taxonomists need to evaluate the optimal balance between the number of diagnostic bases and their stability subject to the topology. In general, pure diagnostic bases rather than private or combined ones should be selected, and such single pure CAs should be compared against all the potentially closely related lineages, not only against the direct sister in a predefined tree entered in CAOS as is the default procedure. We also wish to highlight the following considerations. 1) When basing a species description on molecular data the same rules as in traditional taxonomy should be applied considering deposition and accessibility of data; DNA aliquots and additional type material should be deposited in long term storage facilities, and sequences in public databases (GenBank). As with morphological type specimens, special attention should be given to the storage and availability of molecular types. 2) Due to the underlying homology assumption, we consider the quality of the alignment as critical to determining and extracting diagnostic bases. Thus, we recommend exploring changes to the alignment and, thus, the identified diagnostic characters by applying different alignment programs and masking options. 3) Alignments may change when new data is added, especially concerning non-coding markers. For better traceability, we regard it as beneficial to report not only the alignment position but also refer to a closely related reference genome (if applicable) and report the position in a deposited reference sequence (ideally generated from type material). In its current stage of development, the extraction of diagnostic characters for molecular taxonomy is not yet ready for inclusion in automated species delimitation procedures, as it still requires time-consuming manual steps. However, little adaptation of existing programs would be needed to make them serve molecular taxonomy in its entirety, to overcome the current gap between species discovery and species description.

\section{Methods}

\section{Type localities and collecting sites}

The collecting sites of material included in the present study are shown in Figure 4 (modified after Jörger et al. [25]). Of the three valid species, we were able to recollect $P$. milaschewitchii from its type locality. P. verrucosa was collected in vicinity of the type locality on Guadalcanal, Solomon Islands. Despite several attempts, we were unsuccessful in recollecting $P$. brasilensis at the type locality (see Discussion for assignment of specimens to this species).

\section{Morphology and microanatomy}

Jörger et al. [25] analyzed the radulae of most of the species described above. Unfortunately, for Pontohedyle neridae sp. nov., P. martynovi sp. nov. and P. yurihookeri sp. nov. radulae could not be recovered from the specimens used for DNA extraction. The radula of $P$. wiggi sp. nov. could only be studied under the light microscope, but was lost when attempting to transfer it to a SEM-stub.

Phylogenetic analyses by Jörger et al. [25] revealed two major clades within Pontohedyle. One includes P. milas chewitchii, for which detailed microanatomical and ultrastructural data is available $[55,111]$. The other clade is morphologically poorly characterized, since the original description of $P$. verrucosa lacks details on major organ systems like the reproductive system and the nervous system. For detailed histological comparison of the two major Pontohedyle clades, glutaraldehyde fixed specimens of $P$. 
verrucosa (from near the type locality WP-3 and WP-2 see [25]) were post-fixed in buffered $1 \%$ osmium tetroxide, decalcified using ascorbic acid and embedded in Spurr low-viscosity epoxy resin [112] or Epon epoxy resin (for detailed protocols see $[113,114]$ ). Serial semithin sections ( 1 and $1.5 \mu \mathrm{m})$ of three specimens were prepared using a diamond knife (Histo Jumbo, Diatome, Switzerland) with contact cement on the lower cutting edge to form ribbons [115]. Ribbons were stained using methylene-blue azur II [116] and sealed with Araldit resin under cover slips. Sectioned series are deposited at the Bavarian State Collection of Zoology, Mollusca section (ZSM Mol-20071833, 20071837 and 20100548). Additionally, histological series of Pontohedyle kepii sp. nov. were sectioned as described above.

Digital photographs of each section were taken using a ProgRes C3 camera (Jenoptik, Germany) mounted on a Leica DMB-RBE microscope (Leica Microsystems, Germany). Subsequently, photographs were edited (i.e., grey-scale converted, contrast enhanced and reduced in size) using standard imaging software, then loaded into AMIRA 5.2 (Visage Imaging Software, Germany) for 3D reconstruction of the major organ systems. Alignment, labeling of the organ systems and surface rendering followed in principle the method described by Ruthensteiner [115].

\section{Acquisition of molecular data}

This study aims to characterize the genus Pontohedyle (Acochlidia, Microhedylacea) based on molecular standard markers, i.e., nuclear $18 \mathrm{~S}$ and $28 \mathrm{~S}$ rRNA and mitochondrial COI and 16S rRNA. We included the three previously valid Pontohedyle species (for taxonomy see $[69,83]$ ): P. milaschewitchii (Kowalewsky, 1901), P. verrucosa (Challis, 1970) and recently re-established P. brasilensis (Rankin, 1979) [25]. The nine additional species earlier identified as candidates in the genus Pontohedyle [25] are subject to molecular taxonomy. 28S rRNA, 16S rRNA and COI sequences analyzed by Jörger et al. [25] were retrieved from GenBank (see Table 1 for accession numbers). Additionally, we amplified nuclear $18 \mathrm{~S}$ rRNA (approx. $1800 \mathrm{bp)}$ for at least one individual per species. 18S rRNA was amplified in three parts using the primers for euthyneuran gastropods by Vonnemann et al. [65] and Wollscheid \& Wägele [117]: 18A1 (5' - CCT ACT TCT GGT TGA TCC TGC CAG T $-3^{\prime}$ ), 700R (5' - CGC GGC TGC TGG CAC CAG AC - 3'), 470 F (5' - CAG CAG GCA CGC AAA TTA CCC - 3'), 1500R (5' - CAT CTA GGG CAT CAC AGA CC - 3'), 1155 F (5' - CTG AAA CTT AAA GGA ATT GAC GG 3'), 1800 (5' - TAA TGA TCC TTC CGC AGG TT - 3'). Polymerase chain reactions were conducted using Phire polymerase (New England Biolabs) following this protocol: $98^{\circ} \mathrm{C} 30 \mathrm{sec}, 30-35 \mathrm{x}\left(98^{\circ} \mathrm{C} 5 \mathrm{sec}, 55-65^{\circ} \mathrm{C} 5 \mathrm{sec}, 72^{\circ} \mathrm{C}\right.$
$20-25 \mathrm{sec}), 72^{\circ} \mathrm{C} 60 \mathrm{sec}$. Successful PCR products were cleaned up with ExoSap IT. Cycle sequencing such as sequencing reactions was performed by the Genomic Service Unit (GSU) of the Department of Biology, Ludwig-Maximilians-University Munich, using Big Dye 3.1 kit and an ABI 3730 capillary sequencer. Sequences were edited (forward and reverse strands), concatenated and checked for potential contamination via BLAST searches [108] against the GenBank database via Geneious 5.5.2 [104].

\section{Detection of diagnostic molecular characters}

We used the Characteristic Attribute Organization System (CAOS) $[51,57,58]$ to detect discrete nucleotide substitutions on our previously determined candidate species [25]. The program distinguishes single (single nucleotide) vs. compound (set of nucleotides) 'character attributes' (CA) [51]. Both, single and compound CAs can be further divided into pure (present in all members of a clade but absent from all members of another clade) and private CAs (only present in some members of the clade, but absent in members of other clades) [51]. For taxonomic purposes at this stage we consider only 'single pure characters' ( $\mathrm{sPu}$ ) as diagnostic characters for species descriptions (see Discussion). Since some sister group relationships among Pontohedyle species are not well supported (see [25], Figure 1), we chose our diagnostic molecular characters in the sense of unique within the genus Pontohedyle, rather than assigning plesiomorphic or apomorphic polarity to character states of one species in relation to its direct sister species.

As discussed above, the homology assumption presented in the alignment is crucial for the correct detection of diagnostic characters. For quality control, we performed data input into CAOS with alignments derived from three commonly applied alignment programs and critically compared the resulting differences concerning amounts and positions of the sPus. Alignments were generated for each marker individually using MUSCLE [106], Mafft [118,119] and CLUSTAL W [120]. The COI alignment was checked manually, supported by translation into amino acids. Due to difficulties in aligning highly variable parts of rRNA markers, we removed ambiguous parts of the alignment with two different masking programs, Aliscore [121] and GBlocks [107], and compared the respective effects on character selection. After comparison of the various results we chose MUSCLE [106] in combination with GBlocks [107] as the most conservative approach that results in fewer but more reliable diagnostic characters than the other approaches.

Alignments were analyzed and converted between different formats using Geneious 5.6 (Biomatters) [104]. We performed a phylogenetic analysis under a maximum- 
likelihood approach with RAxML 7.2.8 on each individual marker, applying the 'easy and fast way' described in the RAxML 7.0.4 manual to obtain an input tree. For our present study the phylogenetic hypothesis on sister group relationships of the different Pontohedyle species, however, is not relevant: We manipulated the resulting trees in Mesquite [122], generating a single starting file for CAOS for each species and for each marker, with each of the analyzed species successively being sister to all remaining Pontohedyle species. This iterative procedure retrieves diagnostic characters for the node that compares each single species to all its congeners.

The single gene alignments which formed the basis for the selection of diagnostic nucleotides are available in fasta format as Additional material 3-6. Diagnostic nucleotides are reported with positions in the reference alignment. Position 1 of each alignment refers to position 1 after the primer region, which was removed in the alignment. For better traceability, and in the absence of a closely related reference genome, we additionally report the positions within a reference sequence for each species (deposited in GenBank; see Table 1). In the description of our new species these reference sequences are retrieved from the holotype. Diagnostic molecular characters of the genus Pontohedyle in $18 \mathrm{~S}$ and 28S rRNA are diagnosed based on alignments including all available Pontohedyle sequences (Table 1) and representatives of all other acochlidian genera currently available in public databases (see Additional files 1 and 2 for the original alignments in fasta format).

To meet the requirements by the International Code of Zoological Nomenclature (ICZN) [78,79], this article was registered at ZooBank (www.zoobank.org) under the ZooBank Life Science Identifiers (LSIDs): urn:Isid:zoobank.org:pub:4AE75E9C-4303-42CB-AED277266 C8F6601.

\section{Additional files}

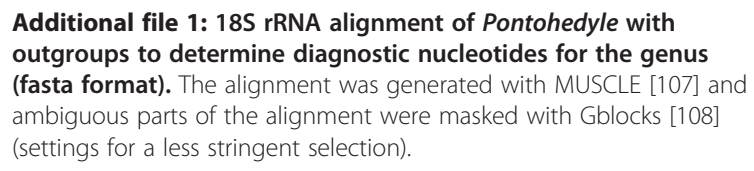

Additional file 2: 28S rRNA alignment of Pontohedyle with outgroups to determine diagnostic nucleotides for the genus (fasta format). The alignment was generated with MUSCLE [107] and ambiguous parts of the alignment were masked with Gblocks [108] (settings for a less stringent selection).

Additional file 3: 18S rRNA alignment of Pontohedyle (fasta format). The alignment was generated with MUSCLE [107] and ambiguous parts of the alignment were masked with Gblocks [108] (settings for a less stringent selection).

Additional file 4: 28S rRNA alignment of Pontohedyle (fasta format). The alignment was generated with MUSCLE [107] and ambiguous parts of the alignment were masked with Gblocks [108] (settings for a less stringent selection).
Additional file 5: 16S rRNA alignment of Pontohedyle (fasta format). The alignment was generated with MUSCLE [107] and ambiguous parts of the alignment were masked with Gblocks [108] (settings for a less stringent selection).

Additional file 6: $\mathrm{COI}$ alignment of Pontohedyle (fasta format). The alignment was generated with MUSCLE [107].

\section{Competing interests}

Both authors declare that they have no competing interests.

\section{Authors' contributions}

KMJ generated the morphological and molecular data and drafted the manuscript. MS planned and supervised the study. The material was collected jointly and in cooperation with a series of collaborators (see Acknowledgements). All authors read and approved the final manuscript.

\section{Acknowledgements}

This study was financed by a WW foundation PhD scholarship to KMJ. We want to thank a series of friends and colleagues who have contributed material and/ or helped with sampling permits: Fontje Kaligis and Gustav Mamangkey (for material from Indonesia), Yuri Hooker (for support in Peru), Peter Ryall and Timea Neusser (for support and material from Ghana), Nerida Wilson and Greg Rouse (for material from Moorea), the organizing team of the World Congress for Malacology 2010 (for sampling permits in Thailand), the Red Sea Environmental Center (for support in collecting material in Egypt), Bastian Brenzinger (for material from Croatia), Sascha Martynov (for material from the Black Sea), the Dumbarton Agricultural Station (for permits in St.Vincent), and Jon Norenburg, Katrine Worsaae, Rick Hochberg and other participants of the Encyclopedia of Life Meiofauna Workshop (for sorting material in the Caribbean). Field activities were supported by DFG SCHR667/ $4,6,9,10$ to MS. The GeoBio Center LMU provided diving equipment. Martin Spies (ZSM) is thanked for valuable discussions and for polishing our English. We would like to express our gratitude to Philippe Bouchet and an anonymous referee for valuable comments on the manuscript.

Received: 2 May 2013 Accepted: 3 September 2013

Published: 27 September 2013

\section{References}

1. Dayrat B: Towards integrative taxonomy. Biol J Linn Soc 2005, 85(3):407-415.

2. Padial JM, Miralles A, De la Riva I, Vences M: The integrative future of taxonomy. Front Zool 2010, 7:16.

3. Padial JM, De La Riva I: A response to recent proposals for integrative taxonomy. Biol J Linn Soc 2010, 101(3):747-756.

4. de Queiroz K: Species concepts and species delimitation. Syst Bio/ 2007, 56(6):879-886.

5. Valdecasas AG, Williams D, Wheeler QD: 'Integrative taxonomy' then and now: a response to Dayrat (2005). Biol J Linn Soc 2008, 93(1):211-216.

6. DeSalle R, Egan MG, Siddall M: The unholy trinity: taxonomy, species delimitation and DNA barcoding. Phil Trans R Soc B 2005, 360(1462):1905-1916.

7. Markmann M, Tautz D: Reverse taxonomy: an approach towards determining the diversity of meiobenthic organisms based on ribosomal RNA signature sequences. Phil Trans R Soc B 2005, 360(1462):1917-1924.

8. Bickford D, Lohman DJ, Sodhi NS, Ng PKL, Meier R, Winker K, Ingram KK, Das I: Cryptic species as a window on diversity and conservation. Trends Ecol Evol 2007, 22(3):148-155.

9. Pfenninger M, Schwenk K: Cryptic animal species are homogeneously distributed among taxa and biogeographical regions. BMC Evol Biol 2007, 7:6.

10. Trontelj P, Fiser C: Cryptic species diversity should not be trivialised. Syst Biodivers 2009, 7(1):1-3.

11. Neusser TP, Jörger KM, Schrödl M: Cryptic species in tropic sands Interactive 3D anatomy, molecular phylogeny and evolution of meiofaunal Pseudunelidae (Gastropoda, Acochlidia). PLOS ONE 2011, 6(8):e23313.

12. Casu M, Curini-Galletti M: Sibling species in interstitial flatworms: a case study using Monocelis lineata (Proseriata: Monocelididae). Mar Biol 2004, 145(4):669-679. 
13. Fontaneto D, lakovenko N, Eyres I, Kaya M, Wyman M, Barraclough TG: Cryptic diversity in the genus Adineta Hudson \& Gosse, 1886 (Rotifera: Bdelloidea: Adinetidae): a DNA taxonomy approach. Hydrobiologia 2011, 662(1):27-33.

14. Fontaneto D, Kaya M, Herniou EA, Barraclough TG: Extreme levels of hidden diversity in microscopic animals (Rotifera) revealed by DNA taxonomy. Mol Phylogenet Evol 2009, 53(1):182-189.

15. Johnson SB, Waren A, Vrijenhoek RC: DNA barcoding of Lepetodrilus limpets reveals cryptic species. J Shellfish Res 2008, 27(1):43-51.

16. Kieneke A, Arbizu PMM, Fontaneto D: Spatially structured populations with a low level of cryptic diversity in European marine Gastrotricha. Mol Ecol 2012, 21(5):1239-1254

17. Puillandre N, Cruaud C, Kantor Yl: Cryptic species in Gemmuloborsonia (Gastropoda: Conoidea). J Molluscan Stud 2010, 76:11-23.

18. Tulchinsky A, Norenburg J, Turbeville J: Phylogeography of the marine interstitial nemertean Ototyphlonemertes parmula (Nemertea, Hoplonemertea) reveals cryptic diversity and high dispersal potential. Mar Biol 2012, 159(3):661-674.

19. Weigand AM, Jochum A, Slapnik R, Schnitzler J, Zarza E, Klussmann-Kolb A: Evolution of microgastropods (Ellobioidea, Carychiidae): integrating taxonomic, phylogenetic and evolutionary hypotheses. BMC Evol Biol 2013, 13(18):1471-2148.

20. Vogler AP, Monaghan MT: Recent advances in DNA taxonomy. J Zool Syst Evol Res 2006, 45(1):1-10.

21. Goldstein PZ, DeSalle R: Integrating DNA barcode data and taxonomic practice: Determination, discovery, and description. Bioessays 2011, 33(2):135-147.

22. DeSalle R: Species discovery versus species identification in DNA barcoding efforts: Response to Rubinoff. Conserv Biol 2006, 20(5):1545-1547.

23. Lipscomb D, Platnick N, Wheeler Q: The intellectual content of taxonomy: a comment on DNA taxonomy. Trends Ecol Evol 2003, 18(2):65-66.

24. Avise JC: Phylogeography: The history and formation of species. Cambridge: Harvard University Press; 2000.

25. Jörger KM, Norenburg JL, Wilson NG, Schrödl M: Barcoding against a paradox? Combined molecular species delineations reveal multiple cryptic lineages in elusive meiofaunal sea slugs. BMC Evol Biol 2012, 12:245.

26. Albano $P G$, Sabelli $B$, Bouchet $P$ : The challenge of small and rare species in marine biodiversity surveys: microgastropod diversity in a complex tropical coastal environment. Biodivers Conserv 2011, 20 (13):3223-3237.

27. Bouchet $P$, Lozouet $P$, Maestrati $P$, Heros V: Assessing the magnitude of species richness in tropical marine environments: exceptionally high numbers of molluscs at a New Caledonia site. Biol I Linn Soc 2002, 75(4):421-436

28. Lim GS, Balke M, Meier R: Determining species boundaries in a world full of rarity: Singletons, species delimitation methods. Syst Biol 2012, 61(1):165-169.

29. Puillandre N, Modica MV, Zhang Y, Sirovich L, Boisselier MC, Cruaud C, Holford M, Samadi S: Large-scale species delimitation method for hyperdiverse groups. Mol Ecol 2012, 21(11):2671-2691.

30. Meyer CP, Paulay G: DNA barcoding: Error rates based on comprehensive sampling. PLoS Biol 2005, 3(12):2229-2238.

31. Hebert PDN, Penton EH, Burns JM, Janzen DH, Hallwachs W: Ten species in one: DNA barcoding reveals cryptic species in the neotropical skipper butterfly Astraptes fulgerator. Proc Natl Acad Sci USA 2004, 101(41):14812-14817

32. Sauer J, Hausdorf B: A comparison of DNA-based methods for delimiting species in a Cretan land snail radiation reveals shortcomings of exclusively molecular taxonomy. Cladistics 2012, 28(3):300-316.

33. Meier R, Shiyang K, Vaidya G, Ng PKL: DNA barcoding and taxonomy in diptera: A tale of high intraspecific variability and low identification success. Syst Biol 2006, 55(5):715-728.

34. Puillandre N, Lambert A, Brouillet S, Achaz G: ABGD, Automatic Barcode Gap Discovery for primary species delimitation. Mol Ecol 2012 21(8):1864-1877.

35. Templeton AR, Crandall KA, Sing CF: A cladistic analysis of phenotypic associations with haplotypes inferred from restriction endonuclease mapping and DNA-sequence data III. Cladogram estimation. Genet 1992, 132(2):619-633.

36. Pons J, Barraclough TG, Gomez-Zurita J, Cardoso A, Duran DP, Hazell S, Kamoun S, Sumlin WD, Vogler AP: Sequence-based species delimitation for the DNA taxonomy of undescribed insects. Syst Bio/ 2006, 55:595-609.

37. Monaghan MT, Wild R, Elliot M, Fujisawa T, Balke M, Inward DJ, Lees DC Ranaivosolo R, Eggleton P, Barraclough TG, et al: Accelerated species inventory on Madagascar using coalescent-based models of species delineation. Syst Biol 2009, 58(3):298-311.

38. Yang ZH, Rannala B: Bayesian species delimitation using multilocus sequence data. Proc Natl Acad Sci U S A 2010, 107(20):9264-9269.

39. Zhang C, Zhang D-X, Zhu T, Yang Z: Evaluation of a Bayesian coalescent method of species delimitation. Syst Biol 2011, 60(6):747-761.

40. Astrin JJ, Stuben PE, Misof B, Wägele JW, Gimnich F, Raupach MJ, Ahrens D: Exploring diversity in cryptorhynchine weevils (Coleoptera) using distance-, character- and tree-based species delineation. Mol Phylogenet Evol 2012, 63(1):1-14

41. Tautz D, Arctander P, Minelli A, Thomas RH, Vogler AP: A plea for DNA taxonomy. Trends Ecol Evol 2003, 18(2):70-74.

42. Blaxter ML: The promise of a DNA taxonomy. Philos Trans R Soc Lond B Biol Sci 2004, 359(1444):669-679.

43. Blaxter M, Elsworth B, Daub J: DNA taxonomy of a neglected animal phylum: an unexpected diversity of tardigrades. Proc $R$ Soc Lond Ser B Biol Sci 2004, 271:S189-S192.

44. Monaghan MT, Balke M, Pons J, Vogler AP: Beyond barcodes: complex DNA taxonomy of a south Pacific island radiation. Proc $R$ Soc B-Biol $\mathrm{SCl}$ 2006, 273(1588):887-893.

45. Monaghan MT, Balke M, Gregory TR, Vogler AP: DNA-based species delineation in tropical beetles using mitochondrial and nuclear markers. Phil Trans R Soc B 2005, 360(1462):1925-1933.

46. Hendrich L, Pons J, Ribera I, Balke M: Mitochondrial cox 1 sequence data reliably uncover patterns of insect diversity but suffer from high lineageidiosyncratic error rates. PLOS ONE 2010, 5(12):e14448.

47. Blaxter M, Mann J, Chapman T, Thomas F, Whitton C, Floyd R, Abebe E: Defining operational taxonomic units using DNA barcode data. Phil Trans R Soc B 2005, 360(1462):1935-1943.

48. Creer S, Fonseca VG, Porazinska DL, Giblin-Davis RM, Sung W, Power DM, Packer M, Carvalho GR, Blaxter ML, Lambshead PJD, et al: Ultrasequencing of the meiofaunal biosphere: practice, pitfalls and promises. Mol Ecol 2010, 19:4-20

49. Brower AVZ: Alleviating the taxonomic impediment of DNA barcoding and setting a bad precedent: names for ten species of 'Astraptes fulgerator' (Lepidoptera: Hesperiidae: Eudaminae) with DNA-based diagnoses. Syst Biodivers 2010, 8(4):485-491.

50. Bauer AM, Parham JF, Brown RM, Stuart BL, Grismer L, Papenfuss TJ, Böhme W, Savage JM, Carranza S, Grismer JL, et al: Availability of new Bayesiandelimited gecko names and the importance of character-based species descriptions. Proc R Soc B Bio/ Sci 2011, 278(1705):490-492.

51. Sarkar IN, Planet PJ, Desalle R: CAOS software for use in character-based DNA barcoding. Mol Ecol Resour 2008, 8(6):1256-1259.

52. Zou SM, Li Q, Kong LF, Yu H, Zheng XD: Comparing the usefulness of distance, monophyly and character-based DNA barcoding methods in species identification: A case study of Neogastropoda. PLOS ONE 2011, 6(10).

53. Challis DA: Hedylopsis cornuta and Microhedyle verrucosa, two new Acochlidiacea (Mollusca: Opisthobranchia) from the Solomon Islands Protectorate. Trans R Soc NZ 1970, 12:29-40.

54. Eder B, Schrödl M, Jörger KM: Systematics and redescription of the European meiofaunal slug Microhedyle glandulifera (Kowalevsky, 1901) (Heterobranchia: Acochlidia): evidence from molecules and morphology. J Molluscan Stud 2011, 77:388-400.

55. Jörger KM, Neusser TP, Haszprunar G, Schrödl M: Undersized and underestimated: 3D-visualization of the Mediterranean interstitial acochlidian gastropod Pontohedyle milaschewitchii (Kowalevsky, 1901). Org Divers Evol 2008, 8:194-214.

56. Jörger KM, Neusser TP, Schrödl M: Re-description of a female Pontohedyle brasilensis (Rankin, 1979), a junior synonym of the Mediterranean $P$. milaschewitchii (Kowalevsky, 1901) (Acochlidia, Gastropoda). Bonn Zool Beitr 2007, 55(3-4):283-290

57. Sarkar IN, Thornton JW, Planet PJ, Figurski DH, Schierwater B, DeSalle R: An automated phylogenetic key for classifying homeoboxes. Mol Phylogenet Evol 2002, 24(3):388-399.

58. Bergmann T, Hadrys H, Breves G, Schierwater B: Character-based DNA barcoding: a superior tool for species classification. Berl Muench Tieraerztl Wochenschr 2009, 122(11-12):446-450. 
59. Odhner N: Die Acochlidiaceen, eine eigentümliche OpisthobranchiatenGruppe. Basteria 1938, 3(1):5-11.

60. Golikov AN, Starobogatov YI: Classis gastropod mollusks - Gastropoda Cuvier, 1797 vol. 3. In Guide on the Black Sea and the Sea of Azove fauna Free living invertebrates. Edited by Vodyanitsky VA. Kyiv: Naukova dumka; 1972:65-166.

61. Kowalevsky A: Les Hédylidés, étude anatomique. Mem Acad Imp Sci StPetersbourg 1901, 12(8):1-32.

62. Rankin JJ: A freshwater shell-less Mollusc from the Caribbean: structure, biotics and contribution to a new understanding of the Acochlidioidea. R Ont Mus Life Sci Contrib 1979, 116:1-123.

63. Jörger KM, Stöger I, Kano Y, Fukuda H, Knebelsberger T, Schrödl M: On the origin of Acochlidia and other enigmatic euthyneuran gastropods, with implications for the systematics of Heterobranchia. BMC Evol Biol 2010, 10:323.

64. Neusser TP, Fukuda H, Jörger KM, Kano Y, Schrödl M: Sacoglossa or Acochlidia? 3D reconstruction, molecular phylogeny and evolution of Aitengidae (Gastropoda: Heterobranchia). J Molluscan Stud 2011, 77:332-350

65. Vonnemann V, Schrödl M, Klussmann-Kolb A, Wägele H: Reconstruction of the phylogeny of the Opisthobranchia (Mollusca: Gastropoda) by means of $18 \mathrm{~S}$ and $28 \mathrm{~S}$ rRNA gene sequences. J Molluscan Stud 2005, 71:113-125.

66. Göbbeler K, Klussmann-Kolb A: Molecular phylogeny of the Euthyneura (Mollusca, Gastropoda) with special focus on Opisthobranchia as a framework for reconstruction of evolution of diet. Thalassas 2011, 27(2):121-154.

67. Wawra E: Geschlechtsdimorphismus bei Pontohedyle milaschewitchii (Kowalevsky, 1901) (Gastropoda: Opisthobranchia). Ann Nathist Mus Wien Ser B Bot Zool 1986, 87(B):325-329.

68. Wawra E: Mitteilung über eine Aufsammlung interstitieller Gastropoden aus sublitoralen Sanden der Insel Lesbos (Griechenland). Ann Nathist Mus Wien Ser B Bot Zool 1974, 78:499-503.

69. Wawra E: Zur Anatomie einiger Acochlidia (Gastropoda, Opisthobranchia) mit einer vorläufigen Revision des Systems und einem Anhang über Platyhedylidae (Opisthobranchia, Ascoglossa). Wien: Universität Wien; 1987.

70. Challis DA: An ecological account of the marine interstitial opisthobranchs of the British Solomon Islands Protectorate. Phil Trans Roy Soc B 1969, 255:527-539.

71. Marcus E: Über Philinoglossacea und Acochlidiacea. Kiel Meeresforsch 1954, 10(2):215-223

72. Marcus E: Three brazilian sand-Opisthobranchia. Bol Fac Filos Ci Letr Univ Sao Paulo, Zool 1953, 164(18):165-203.

73. Curini-Galletti M, Artois T, Delogu V, De Smet WH, Fontaneto D, Jondelius U, Leasi F, Martínez A, Meyer-Wachsmuth I, Nilsson KS, et al: Patterns of diversity in soft-bodied meiofauna: Dispersal ability and body size matter. PLOS ONE 2012, 7(3):e33801.

74. Leasi F, Todaro M: Meiofaunal cryptic species revealed by confocal microscopy: the case of Xenotrichula intermedia (Gastrotricha). Mar Biol 2009, 156(6):1335-1346.

75. Krug PJ, Vendetti JE, Rodriguez AK, Retana JN, Hirano YM, Trowbridge CD: Integrative species delimitation in photosynthetic sea slugs reveals twenty candidate species in three nominal taxa studied for drug discovery, plastid symbiosis or biological control. Mol Phylogenet Evol 2013. http://dx.doi.org/10.1016/j.ympev.2013.0 (in press).

76. Westheide W, Schmidt H: Cosmopolitan versus cryptic meiofaunal polychaete species: an approach to a molecular taxonomy. Helgol Mar Res 2003, 57(1):1-6.

77. Cook LG, Edwards RD, Crisp MD, Hardy NB: Need morphology always be required for new species descriptions? Invertebr Syst 2010, 24(3):322-326.

78. Int Commission Z: Amendment of Articles 8, 9, 10, 21 and 78 of the International Code of Zoological Nomenclature to expand and refine methods of publication. Zootaxa 2012, 3450:1-7.

79. Nomenclature ICoZ: International code of zoological nomenclature. 4th edition. London: International Trust for zoological Nomenclature; 1999

80. Brenzinger B, Neusser TP, Glaubrecht M, Haszprunar G, Schrödl M: Redescription and three-dimensional reconstruction of the limnic acochlidian gastropod Strubellia paradoxa (Strubell, 1892) (Gastropoda: Euthyneura) from Ambon, Indonesia. J Nat Hist 2011, 45(3-4):183-209.

81. Neusser TP, Heß M, Schrödl M: Tiny but complex - interactive 3D visualization of the interstitial acochlidian gastropod Pseudunela cornuta (Challis, 1970). Front Zool 2009, 6(1):20.
82. Neusser TP, Martynov AV, Schrödl M: Heartless and primitive? 3D reconstruction of the polar acochlidian gastropod Asperspina murmanica. Acta Zool (Stock) 2009, 90:228-245.

83. Schrödl M, Neusser TP: Towards a phylogeny and evolution of Acochlidia (Mollusca: Gastropoda: Opisthobranchia). Zool J Linn Soc 2010, 158:124-154

84. Schlick-Steiner BC, Seifert B, Stauffer C, Christian E, Crozier RH, Steiner FM: Without morphology, cryptic species stay in taxonomic crypsis following discovery. Trends Ecol Evol 2007, 22(8):391-392.

85. Leaché AD, Fujita MK: Bayesian species delimitation in West African forest geckos (Hemidactylus fasciatus). Proc R Soc B Biol Sci 2010, 277(1697):3071-3077.

86. Fujita MK, Leache AD, Burbrink FT, McGuire JA, Moritz C: Coalescent-based species delimitation in an integrative taxonomy. Trends Ecol Evol 2012, 27(9):480-488.

87. Fujita MK, Leaché AD: A coalescent perspective on delimiting and naming species: a reply to Bauer et al. Proc R Soc B Biol Sci 2011, 278(1705):493-495.

88. Rach J, DeSalle R, Sarkar IN, Schierwater B, Hadrys H: Character-based DNA barcoding allows discrimination of genera, species and populations in Odonata. Proc R Soc B Biol Sci 2008, 275(1632):237-247.

89. Pleijel F, Jondelius U, Norlinder E, Nygren A, Oxelman B, Schander C, Sundberg P, Thollesson M: Phylogenies without roots? A plea for the use of vouchers in molecular phylogenetic studies. Mol Phylogenet Evol 2008, 48(1):369-371

90. De Ley P, De Ley IT, Morris K, Abebe E, Mundo-Ocampo M, Yoder M, Heras J, Waumann D, Rocha-Olivares A, Burr AHJ, et al: An integrated approach to fast and informative morphological vouchering of nematodes for applications in molecular barcoding. Phil Trans R SOC B 2005, 360(1462):1945-1958.

91. Geiger DL, Thacker CE: Micromolluscs in molecular systematics: Experiences and best practices. In Micromolluscs: Methodological Challenges - Exciting Results: Proceedings from the Micromollusc Symposium of the 16th Unitas Malacologica World Congress of Malacology. Edited by Geiger DL, Ruthensteiner B. Zoosymposia, Magnolia Press; 2008:39-45.

92. Bouchet $P$, Strong EE: Historical name-bearing types in marine molluscs: an impediment to biodiversity studies? In Systema Naturae. Edited by Polaszek A. London: CRC Press; 2010:63-74.

93. Hajibabaei M, Smith MA, Janzen DH, Rodriguez JJ, Whitfield JB, Hebert PDN: A minimalist barcode can identify a specimen whose DNA is degraded. Mol Ecol Notes 2006, 6(4):959-964.

94. Kadereit G, Piirainen M, Lambinon J, Vanderpoorten M: Cryptic taxa should have names: Reflections in the glasswort genus Salicornia (Amaranthaceae). Taxon 2012, 61(6):1227-1239.

95. Blanchette M, Diallo AB, Green ED, Miller W, Haussler D: Computational reconstruction of ancestral DNA sequences. Methods Mol Biol 2008 422:171-184.

96. Reid BN, Le M, McCord WP, Iverson JB, Georges A, Bergmann T, Amato G, Desalle R, Naro-Maciel E: Comparing and combining distance-based and character-based approaches for barcoding turtles. Mol Ecol Resour 2011, 11(6):956-967.

97. Kvist S, Sarkar IN Erseus C: Genetic variation and phylogeny of the cosmopolitan marine genus Tubificoides (Annelida: Clitellata: Naididae: Tubificinae). Mol Phylogenet Evol 2010, 57(2):687-702.

98. Andriaholinirina N, Fausser JL, Roos C, Zinner D, Thalmann U, Rabarivola C, Ravoarimanana I, Ganzhorn JU, Meier B, Hilgartner R, et al: Molecular phylogeny and taxonomic revision of the sportive lemurs. BMC Evol Biol 2006, 6:6.

99. Gittenberger A, Gittenberger E: Cryptic, adaptive radiation of endoparasitic snails: sibling species of Leptoconchus (Gastropoda: Coralliophilidae) in corals. Org Divers Evol 2011, 11(1):21-41.

100. Ornelas-Gatdula E, Camacho-García Y, Schrödl M, Padula V, Hooker Y, Gosliner TM, Valdés Á: Molecular systematics of the 'Navanax aenigmaticus' species complex (Mollusca, Cephalaspidea): coming full circle. Zool Scr 2012, 41(4):374-385.

101. Geiger MF, Schliewen UK: Gymnocephalus ambriaelacus, a new species of ruffe from Lake Ammersee, southern Germany (Teleostei, Perciformes, Percidae). Spixiana 2010, 33(1):119-137.

102. Edgecombe GD, Giribet G: A New Zealand species of the trans-Tasman centipede order Craterostigmomorpha (Arthropoda : Chilopoda) corroborated by molecular evidence. Invertebr Syst 2008, 22(1):1-15. 
103. Yassin A, Markow TA, Narechania A, O'Grady PM, DeSalle R: The genus Drosophila as a model for testing tree- and character-based methods of species identification using DNA barcoding. Mol Phylogenet Evol 2010, 57(2):509-517.

104. Drummond A, Ashton B, Buxton S, Cheung M, Cooper A, Heled J, Kearse M, Moir R, Stones-Havas S, Strurrock S, et al: Geneious v5.4, In; 2010. www. geneious.com

105. Chakrabarty P: Genetypes: a concept to help integrate molecular phylogenetics and taxonomy. Zootaxa 2010, 2632:67-68.

106. Edgar RC: MUSCLE: multiple sequence alignment with high accuracy and high throughput. Nucleic Acids Res 2004, 32(5):1792-1797.

107. Talavera G, Castresana J: Improvement of phylogenies after removing divergent and ambiguously aligned blocks from protein sequence alignments. Syst Biol 2007, 56(4):564-577.

108. Altschul SF, Gish W, Miller W, Myers EW, Lipman DJ: Basic local alignment search tool. J Mol Biol 1990, 215(3):403-410.

109. Haszprunar G: Species delimitations - not 'only descriptive'. Org Divers Evol 2011, 11(3):249-252.

110. Wheeler QD: Taxonomic triage and the poverty of phylogeny. Philos Trans R Soc Lond B Biol Sci 2004, 359(1444):571-583.

111. Jörger KM, Heß M, Neusser TP, Schrödl M: Sex in the beach: spermatophores, dermal insemination and 3D sperm ultrastructure of the aphallic mesopsammic Pontohedyle milaschewitchii (Acochlidia, Opisthobranchia, Gastropoda). Mar Biol 2009, 156(6):1159-1170.

112. Spurr AR: A low-viscosity epoxy resin embedding medium for electron microscopy. J Ultrastruc Res 1969, 26:31-43.

113. Neusser TP, Heß M, Haszprunar G, Schrödl M: Computer-based threedimensional reconstruction of the anatomy of Microhedyle remanei (Marcus, 1953), an interstitial acochlidian gastropod from Bermuda. J Morphol 2006, 267(2):231-247.

114. Neusser TP, Schrödl M: Tantulum elegans reloaded: a computer-based 3D-visualization of the anatomy of a Caribbean freshwater acochlidian gastropod. Invertebr Biol 2007, 126(1):18-39.

115. Ruthensteiner B: Soft Part 3D visualization by serial sectioning and computer reconstruction. Zoosymposia 2008, 1:63-100.

116. Richardson KC, Jarett L, Finke EH: Embedding in epoxy resins for ultrathin sectioning in electron microscopy. Stain Technol 1960, 35:313-323.

117. Wollscheid $E$, Wägele $H$ : Initial results on the molecular phylogeny of the Nudibranchia (Gastropoda, Opisthobranchia) based on 18S rDNA data. Mol Phylogenet Evol 1999, 13(2):215-226.

118. Katoh K, Kuma K, Toh H, Miyata T: MAFFT version 5: improvement in accuracy of multiple sequence alignment. Nucleic Acids Res 2005, 33(2):511-518.

119. Katoh K, Misawa K, Kuma K, Miyata T: MAFFT: a novel method for rapid multiple sequence alignment based on fast Fourier transform. Nucleic Acids Res 2002, 30(14):3059-3066.

120. Chenna R, Sugawara H, Koike T, Lopez R, Gibson TJ, Higgins DG, Thompson JD: Multiple sequence alignment with the Clustal series of programs. Nucleic Acids Res 2003, 31(13):3497-3500.

121. Misof B, Misof K: A Monte Carlo approach successfully identifies randomness in multiple sequence alignments: A more objective means of data exclusion. Syst Biol 2009, 58(1):21-34.

122. Maddison DR: Mesquite: a modular system for evolutionary analysis. Version 2.75; 2011. http://mesquiteproject.org.

\section{Submit your next manuscript to BioMed Central and take full advantage of:}

- Convenient online submission

- Thorough peer review

- No space constraints or color figure charges

- Immediate publication on acceptance

- Inclusion in PubMed, CAS, Scopus and Google Scholar

- Research which is freely available for redistribution 